\title{
Non-alcoholic fatty liver disease and its relationship with cardiovascular disease and other extrahepatic diseases
}

\author{
Leon A Adams, ${ }^{1}$ Quentin M Anstee, ${ }^{2,3}$ Herbert Tilg, $^{4}$ Giovanni Targher ${ }^{5}$
}

\begin{abstract}
'School of Medicine and Pharmacology, The University of Western Australia, Nedlands, Western Australia, Australia ${ }^{2}$ Faculty of Medical Sciences, Institute of Cellular Medicine, Newcastle University, Newcastle Upon Tyne, UK

${ }^{3}$ Liver Unit, Newcastle Upon Tyne Hospitals NHS Trust,

Freeman Hospital, Newcastle upon Tyne, UK

${ }^{4}$ Department of Internal Medicine I, Gastroenterology, Hepatology \& Metabolism, Medical University Innsbruck, Innsbruck, Austria

${ }^{5}$ Division of Endocrinology, Diabetes and Metabolism, Department of Medicine, University and Azienda Ospedaliera Universitaria Integrata of Verona, Verona, Italy
\end{abstract}

\section{Correspondence to} Professor Giovanni Targher, Section of Endocrinology, Diabetes and Metabolism, Department of Medicine, University and Azienda Ospedaliera Universitaria Integrata of Verona, Piazzale Stefani, 1, Verona 37126, Italy; giovanni.targher@univr.it

LAA and QMA contributed equally.

Received 1 February 2017 Revised 14 February 2017 Accepted 16 February 2017 Published Online First 17 March 2017

CrossMark

To cite: Adams LA, Anstee QM, Tilg $\mathrm{H}$, et al. Gut 2017;66:1138-1153.

\section{ABSTRACT}

Key physiological functions of the liver, including glucose and lipid metabolism, become disturbed in the setting of non-alcoholic fatty liver disease (NAFLD) and may be associated with a systemic inflammatory 'milieu' initiated in part by liver-secreted cytokines and molecules. Consequently, the pathophysiological effects of NAFLD extend beyond the liver with a large body of clinical evidence demonstrating NAFLD to be independently associated with both prevalent and incident cardiovascular disease (CVD), chronic kidney disease (CKD) and type 2 diabetes mellitus (T2DM). The magnitude of risk of developing these extrahepatic diseases parallels the underlying severity of NAFLD, such that patients with non-alcoholic steatohepatitis (NASH) appear to be at greater risk of incident CVD, CKD and T2DM than those with simple steatosis. Other modifiers of risk may include genetic variants (eg, patatin-like phospholipase domain-containing 3 and trans-membrane 6 superfamily member 2 polymorphisms), visceral adipose tissue accumulation, dietary intake and the gut microbiome. Emerging data also suggest that NAFLD may be a risk factor for colonic neoplasia and reduced bone mineral density, especially among men. Importantly, improvement/resolution of NAFLD is associated with a reduced incidence of T2DM and improved kidney function, adding weight to causality and suggesting liver focused treatments may reduce risk of extrahepatic complications. Awareness of these associations is important for the clinicians such that CVD risk factor management, screening for T2DM and CKD are part of the routine management of patients with NAFLD.

\section{INTRODUCTION}

Non-alcoholic fatty liver disease (NAFLD) is a very common pathological condition worldwide that is closely associated with the clinical features of metabolic syndrome and is characterised by substantial interpatient variability in severity and rate of liver disease progression. ${ }^{12}$

A purely liver-centric view is that NAFLD represents a spectrum of progressive liver disease occurring in the absence of excessive alcohol consumption that ranges from isolated intrahepatic triglyceride accumulation (simple steatosis), through intrahepatic triglyceride accumulation plus inflammation and hepatocyte injury (non-alcoholic steatohepatitis, NASH), and ultimately progresses to fibrosis/cirrhosis and potentially hepatocellular carcinoma. ${ }^{3}$ Although a significant proportion of the population has NAFLD, only a minority progresses to advanced liver disease or liver-related death. ${ }^{3}$
However, this liver-centric view does not encompass the wider ramifications of NAFLD. Indeed, NAFLD is just one facet of a multisystem disease (figure 1) that confers substantially increased morbidity and mortality on those patients who are affected and where the most common causes of death are cardiovascular disease (CVD), followed by extrahepatic malignancies and liver-related complications. $^{45}$

This review mainly focuses on the principle extrahepatic chronic diseases associated with NAFLD where there is now strongest evidence for a potential causal link: CVD, type 2 diabetes mellitus (T2DM), chronic kidney disease $(\mathrm{CKD})^{1}{ }^{2}$ and, to a lesser extent, certain types of extrahepatic tumours and osteoporosis.

\section{EPIDEMIOLOGICAL DATA LINKING NAFLD TO RISK OF CVD}

That NAFLD is associated with an increased risk of CVD is unsurprising, given the close associations between NAFLD and the established CVD risk factors encapsulated by the metabolic syndrome, including abdominal obesity, hypertension, atherogenic dyslipidaemia and insulin resistance/dysglycaemia. $^{6-8}$ Highlighting the intimacy of these associations is the observation that the increases in hepatic lipid accumulation are directly proportional to the severity of each component of the metabolic syndrome. ${ }^{9}$ However, the nature and extent of these associations, whether simply due to a shared underlying aetiology or because the presence of NAFLD confers an additional risk, remains the subject of much scrutiny. This attention is undoubtedly warranted as it has important clinical implications for screening and surveillance strategies in the growing number of patients with NAFLD. Addressing CVD risk in patients with NAFLD is also the aspect of the disease most amenable to medical management and so improving long-term clinical outcomes.

Beyond the associations with traditional CVD risk factors, patients with NAFLD also exhibit a range of non-traditional CVD risk factors, including hyperuricaemia, ${ }^{10-12}$ hypoadiponectinaemia ${ }^{13-15}$ and hypovitaminosis D. ${ }^{16}$ The association with $\mathrm{CKD}$, discussed later in this review, also confers an increased risk of CVD. ${ }^{17}$ Other potentially contributing risk factors include increased levels of circulating proinflammatory markers (eg, $\mathrm{C}$ reactive protein, interleukin (IL) 6, tumour necrosis factor (TNF)- $\alpha$ and other hepatic acute-phase proteins), procoagulant factors (eg, fibrinogen and plasminogen activator inhibitor-1) and adhesion molecules 
Figure 1 Non-alcoholic fatty liver disease (NAFLD): a multisystem disease. Reported associations between NAFLD and various human diseases.

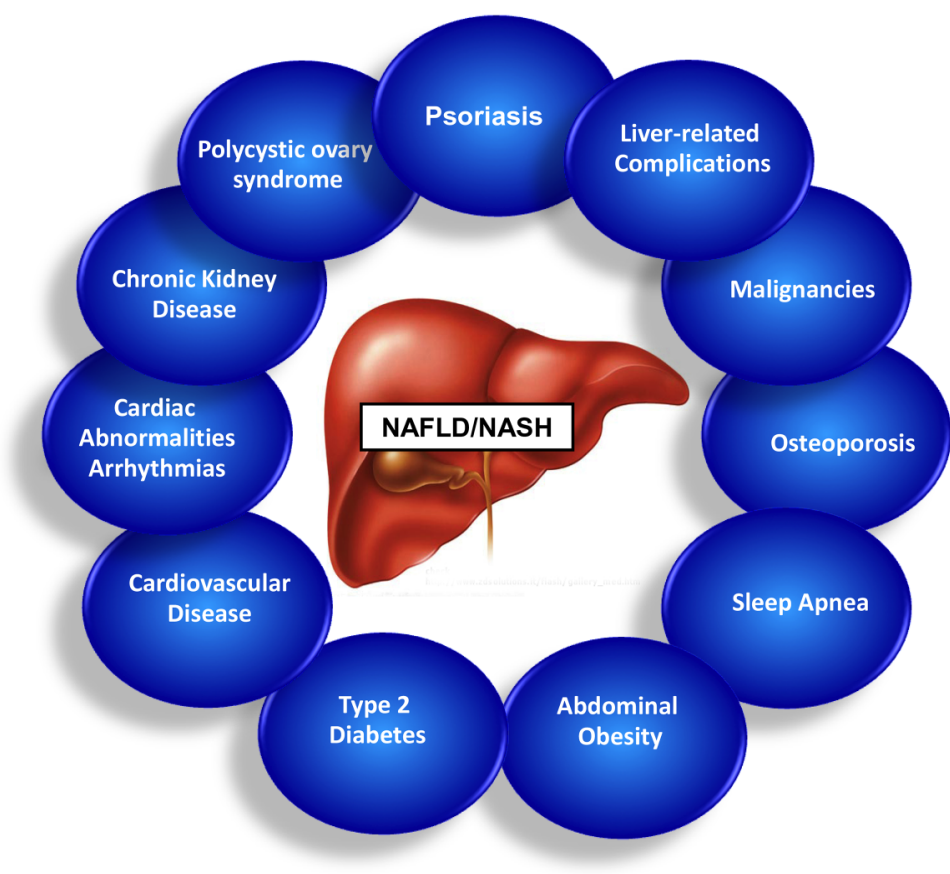

(eg, vascular adhesion protein-1) that are likely to have been synthesised within the liver, especially in the presence of NASH. ${ }^{18-23}$

Substantial epidemiological evidence links NAFLD with objectively assessed subclinical atherosclerosis and with an increased prevalence of clinically manifest CVD. In case-control studies, NAFLD has been linked with increased carotid artery intima-media thickness, ${ }^{24-28}$ increased arterial wall stiffness, ${ }^{29} 30$ and impaired endothelium-dependent flow-mediated vasodilatation. $^{31} 32$ Similarly, a meta-analysis of seven cross-sectional studies totalling 3497 individuals concluded that ultrasounddiagnosed NAFLD was strongly associated with greater carotid-artery intimal medial thickness and an increased prevalence of carotid atherosclerotic plaques. ${ }^{33}$ These findings have since been supported by a larger meta-analysis that incorporated 27 studies and confirmed the association of NAFLD with various markers of subclinical atherosclerosis (including also increased coronary-artery calcium score) independent of traditional CVD risk factors and metabolic syndrome features. ${ }^{34}$ Individuals with NAFLD among an occupational cohort of over 10000 South Korean people had an increased coronary-artery calcium score, independent of multiple CVD risk factors, including insulin resistance. ${ }^{35}$ Case-control studies have also reported strong associations of NAFLD with early changes in left ventricular morphology and/or diastolic dysfunction, ${ }^{36-39}$ impaired myocardial energy metabolism ${ }^{40}{ }^{41}$ and reduced coronary artery flow. ${ }^{42}$ Cohort studies in patients with biopsyconfirmed NAFLD/NASH also clearly demonstrated that CVD is the most common cause of death. ${ }^{45}$

Population-based cohort studies, such as the National Health and Nutrition Examination Survey (NHANES-III), also provide evidence of increased CVD prevalence in NAFLD. In approximately 11500 adult NHANES participants, NAFLD (diagnosed by ultrasonography) remained significantly associated with an increased prevalence of CVD after adjusting for major demographic, clinical and metabolic confounders. ${ }^{43} 44$ An Italian study in nearly 3000 outpatients with T2DM also demonstrated that those with ultrasound-diagnosed NAFLD had a higher prevalence of coronary, cerebrovascular and peripheral vascular disease, independent of traditional CVD risk factors, diabetesrelated variables and other potential confounders. ${ }^{45}$ Among patients undergoing clinically indicated coronary angiography, the presence of NAFLD also independently correlated with the severity of coronary artery disease. ${ }^{46-48}$ Recently, a systematic review and meta-analysis incorporating almost 165000 participants in 34 studies ( 21 cross-sectional, 13 cohort studies) has provided further support for the association of NAFLD (diagnosed by biochemistry, imaging or histology) with atherosclerosis, hypertension and both prevalent and incident CVD. ${ }^{49}$

Based on the available data, there seems little doubt that NAFLD is associated with increased CVD prevalence, an association that has been consistently replicated across different patient populations. Whether NAFLD is an independent CVD risk factor or simply a bystander that shares common aetiological factors remains controversial. ${ }^{3}$ However, there is a growing body of evidence demonstrating that CVD is a clinical concern in NAFLD, and that patients with NAFLD are more likely to experience a CVD-related death than a liver-related death. ${ }^{4} 5$ Although some studies suggested that only patients with NASH rather than those with simple steatosis have an increased CVD mortality compared with the matched control population, ${ }^{50} 51$ a subsequent meta-analysis brought this into question. In that meta-analysis patients with NAFLD (as detected by histology or ultrasonography) had a substantially greater risk of CVD mortality than the matched control population but presence of NASH did not further increase risk of CVD mortality. ${ }^{52}$ Further prospective studies in patients with biopsy-characterised NAFLD are needed to address this point, although some studies with a sufficiently long follow-up recently showed that fibrosis stage rather than $\mathrm{NASH}$ best predicts long-term survival outcomes of patients with NAFLD. ${ }^{4}$

Several studies support an association between NAFLD and incident CVD. Recent retrospective cohort studies reported a significant association between ultrasound-diagnosed NAFLD and the progression of subclinical coronary or carotid 
atherosclerosis independent of multiple CVD risk factors. The risk of subclinical carotid/coronary atherosclerosis progression was also higher among patients with NAFLD with increased non-invasive markers of advanced fibrosis at baseline (NAFLD fibrosis score (NFS), fibrosis-4 score or elevated $\gamma$-glutamyl transferase levels). ${ }^{53}{ }^{54}$ Additionally, the regression of NAFLD on ultrasound over time was associated with a decreased risk of subclinical carotid atherosclerosis development. ${ }^{53}$

The presence of NAFLD at baseline, although defined clinically using the calculated fatty liver index (FLI), conferred an OR of 1.63 for subsequent development of carotid atherosclerotic plaques in a cohort of 1872 European patients. ${ }^{55}$ Recent data also support a bidirectional association between incident NAFLD and CVD risk factors. After controlling for potential confounding factors, including alcohol consumption, in 1051 participants of the Framingham Heart Study, individuals with NAFLD at baseline were more likely to subsequently develop incident hypertension and T2DM than those without NAFLD; conversely, those with metabolic risk factors (hypertriglyceridaemia, hypertension or T2DM) at baseline were more likely to develop incident NAFLD. ${ }^{56}$ These findings are also consistent with natural history studies of patients with NAFLD with serial liver biopsies where those with occurrence of incident T2DM or hypertension between biopsies were most likely to exhibit progressive liver fibrosis. ${ }^{1} 2$ A large number of observational studies also suggest that NAFLD is predictive of incident CVD events and death. ${ }^{4} 5051$ 57-67 Table 1 lists the principal retrospective and prospective studies that have addressed the relationship between NAFLD (defined radiologically or histologically) and risk of CVD events. Many of these studies were also included in an updated meta-analysis that incorporated a total of 16 unique, observational studies with 34043 adults and captured nearly 2600 CVD outcomes over a median of 6.9-year follow-up. ${ }^{68}$ This meta-analysis concluded that the presence of NAFLD (diagnosed by imaging or histology) conferred an OR of 1.64 for fatal and non-fatal incident CVD events, a risk that appeared to increase further with greater severity of NAFLD and remained significant in only those studies where analysis was fully adjusted for potentially confounding covariates. ${ }^{68}$ However, some of these studies have suggested that the increase in CVD risk may be limited to subgroups of patients with NAFLD, such as those with advanced fibrosis, ${ }^{57} \mathrm{~T}^{2} \mathrm{DM}^{58}$ or men with an elevated $\gamma$-glutamyl transferase level. ${ }^{58}$ It is, therefore, conceivable that additional factors may modify the association between NAFLD and risk of CVD events. One such modifying factor may be genetic variation.

Advances in our understanding of how genetic modifiers influence disease progression through genome-wide association studies are also relevant and have provided important insights into the crosstalk between NAFLD and CVD and how they may become dissociated. ${ }^{69}$ Although the widely validated single nucleotide polymorphism, rs738409 (c.444 C>G, p.I148M) in patatin-like phospholipase domain-containing 3 (PNPLA3) confers an increased risk of NASH, hepatic fibrosis and hepatocellular carcinoma, it has no apparent effect on CVD risk. ${ }^{70-72}$ However, further well designed prospective studies are needed to better clarify this controversial issue. ${ }^{70-73}$ Recently, another non-synonymous genetic variant within a gene of unknown function called transmembrane 6 superfamily member 2, TM6SF2 (rs58542926 c.449 C>T, p.E167K), has been linked with NAFLD. ${ }^{74}$ As with PNPLA3, carriage of the TM6SF2 minor $(\mathrm{T})$ allele is associated with greater hepatic steatosis, more severe NASH and greater hepatic fibrosis/cirrhosis, ${ }^{75} 76$ but intriguingly, carriage of the more common major (C) allele promotes very low density lipoprotein excretion, conferring an increased risk of dyslipidaemia and CVD. ${ }^{76} 77$ Thus, while in general NAFLD is associated with an increased risk of CVD, carriage of specific genetic modifiers might mean that these may become dissociated. In what has been described as the "catch-22 paradigm', individuals that possess the TM6SF2 minor (T) allele will be more prone to liver-related morbidity, while those that carry the more common (C) allele may be at greater risk of CVD. ${ }^{78}$ Whether the strength of this effect is sufficient to overcome the effects of many other environmental or genetic variants that influence outcome(s) and so be clinically relevant at an individual level does however remain to be definitely determined. ${ }^{78}$ The relatively new field of epigenetics research is also providing exciting insights into the role of DNA methylation in the pathogenesis of NAFLD and how gene-environment interactions may influence CVD. ${ }^{79-82}$

Accumulating evidence also suggests that NAFLD is linked with valvular heart diseases (ie, aortic-valve sclerosis or mitral annulus calcification) and increased risk of cardiac arrhythmias (mainly atrial fibrillation and ventricular tachyarrhythmias) that may partly contribute to explain the increased risk of CVD events observed in patients with NAFLD. ${ }^{83-87}$

Overall, therefore, the available evidence demonstrates the strong association between NAFLD and CVD and supports the view that NAFLD increases CVD risk: a relationship that may be modified by a range of factors including genetic variation. This has important clinical implications that may influence the decision to institute primary prevention strategies with lipid-lowering, antihypertensive or antiplatelet agents. At present, algorithms such as the Framingham risk equation or the Systematic COronary Risk Evaluation (SCORE) risk charts for CVD risk assessment are used to guide initiation of preventative interventions. However, they are based on traditional CVD risk factors and do not include presence of NAFLD. Although the Framingham risk equation has been validated for use in patients with NAFLD, ${ }^{88}$ it remains unclear whether the addition of NAFLD as a predictive factor in this or other CVD risk score systems can improve their ability to accurately predict future CVD events. Moreover, randomised clinical trials with CVD outcomes that focus on treatments for liver disease in NAFLD are also required in order to definitely establish a causal relationship between NAFLD and risk of CVD events.

\section{EPIDEMIOLOGICAL DATA LINKING NAFLD TO RISK OF CKD}

Over the past decade, several population-based and hospitalbased studies have shown that the prevalence of CKD (defined as estimated glomerular filtration rate (eGFR) $<60 \mathrm{~mL} / \mathrm{min} /$ $1.73 \mathrm{~m}^{2}$, abnormal albuminuria or overt proteinuria) was markedly increased in patients with NAFLD as diagnosed by imaging $^{89-98}$ or histology. ${ }^{99-103}$ In these studies, the prevalence of CKD ranged from approximately $20 \%$ to $55 \%$ among patients with NAFLD compared with $5 \%$ to $30 \%$ among those without NAFLD. ${ }^{89-103}$ Notably, in most of these studies the significant association between NAFLD and CKD persisted after adjustment for hypertension, T2DM and other known risk factors for CKD. Pan et al recently reported that after adjustment for visceral fat accumulation and other cardiometabolic risk factors, there was a positive, graded relationship between intrahepatic triglyceride content, as measured by magnetic resonance spectroscopy, and the presence of CKD or abnormal albuminuria in obese individuals. ${ }^{97}$ In a population-based study of 8270 Chinese adults with normal kidney function, NAFLD was independently associated with low levels of albuminuria also, defined as urine albumin/creatinine ratio below $30 \mathrm{mg} /$ 


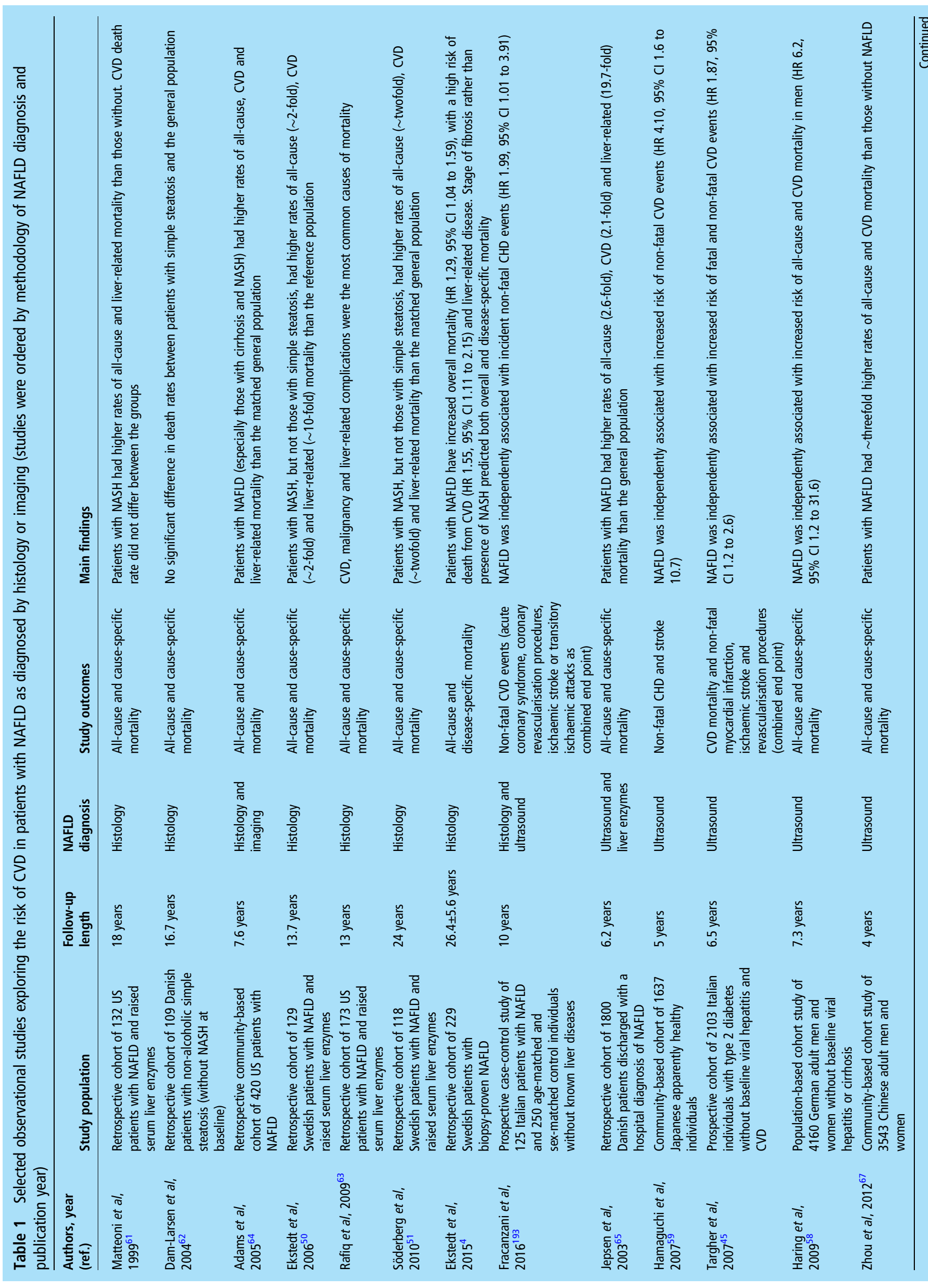




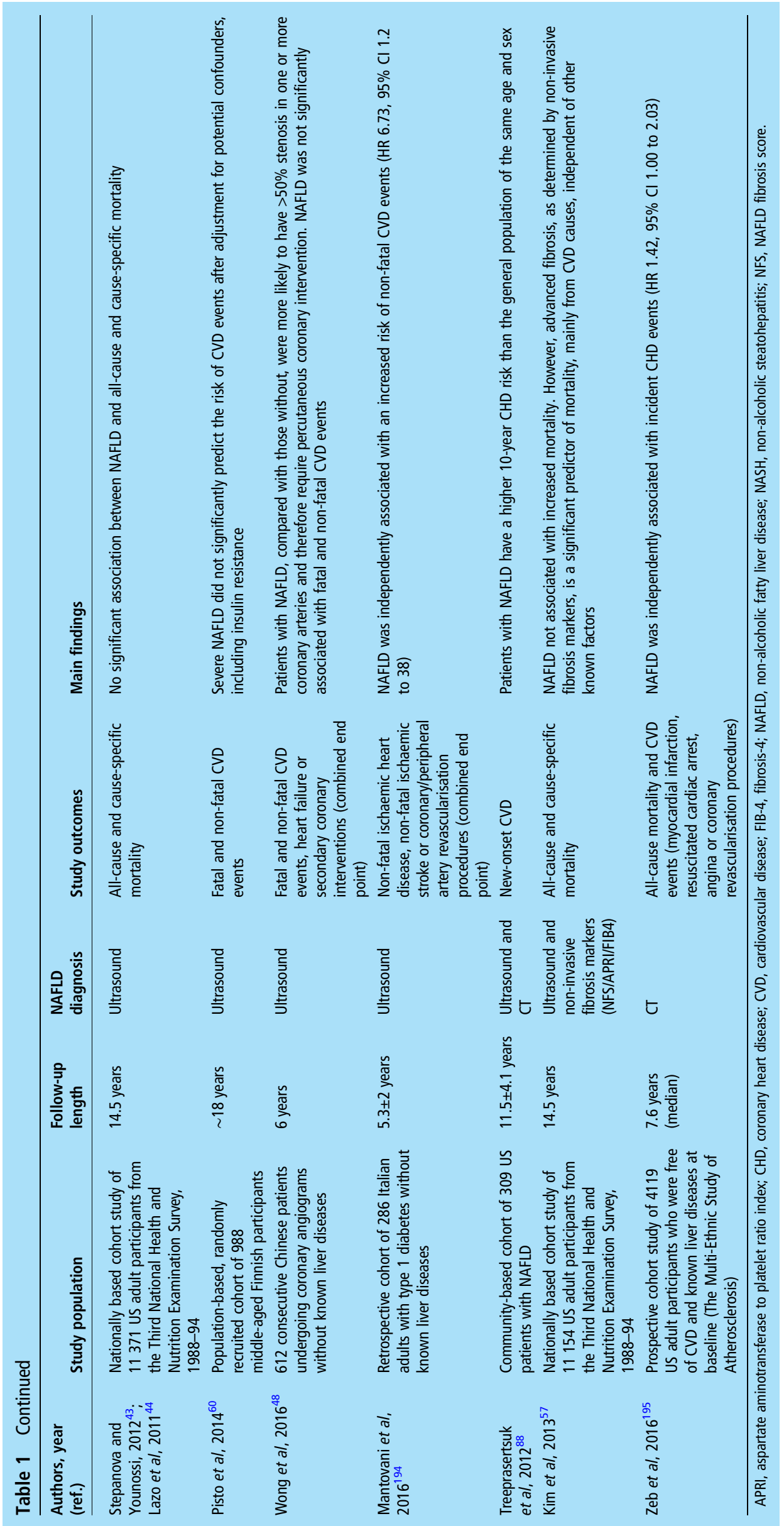


g. ${ }^{104}$ The finding of a strong association between NAFLD and early kidney dysfunction (ie, microalbuminuria or eGFR $<90 \mathrm{~mL} / \mathrm{min} / 1.73 \mathrm{~m}^{2}$ ) has been also documented in a cohort of nearly 600 overweight or obese children. ${ }^{98}$ Recognition of this kidney dysfunction in childhood is clinically important because the treatment to reverse it is more likely to be effective if applied early in the disease process. Interestingly, some casecontrol studies that used liver biopsy to diagnose NAFLD also reported a significant, graded relationship between the severity of NAFLD histology and the presence of either decreased eGFR or abnormal albuminuria. ${ }^{99-103}$ For example, in a case-control study, we found that patients with biopsy-confirmed NASH had a greater prevalence of abnormal albuminuria and CKD than matched control subjects without hepatic steatosis, and that the histological severity of NASH was independently associated with decreasing mean eGFR values (figure 2). ${ }^{99}$ That said, it is important to underline that none of the abovementioned studies have used renal biopsy to examine the pathology of the CKD; so, it remains currently unknown if NAFLD is associated with a specific type of kidney disease (though it is very likely that NAFLD contributes to kidney damage mainly through accelerated atherogenesis). ${ }^{89-103}$

Although the cross-sectional associations between NAFLD and CKD stages are strong and consistent across a wide range of patient populations, the data on whether NAFLD per se is causally linked to the development and progression of CKD remain debateable. $^{105106}$

In an occupational cohort of nearly 8500 non-diabetic and non-hypertensive South Korean men with preserved kidney function and no overt proteinuria at baseline who were followed up for a mean period of 3.2 years, NAFLD (diagnosed by ultrasonography) was associated with an increased incidence of CKD (HR $1.60 ; 95 \%$ CI 1.3 to 2.0$)^{107}$ This association remained significant after adjusting for body mass index (BMI), blood pressure, insulin resistance, plasma $\mathrm{C}$ reactive protein and other potential confounding factors. ${ }^{107}$ Similarly, in the Valpolicella Heart Diabetes Study, involving 1760 patients with type 2 diabetes with preserved kidney function who were followed up over a 6.5-year period, the presence of NAFLD on ultrasonography was associated with an increased incidence of CKD (HR 1.69 ; $95 \%$ CI 1.3 to 2.6 ) independently of traditional cardiorenal risk factors, diabetes-related variables and medication use. ${ }^{108}$ Consistent with these findings, in a longitudinal cohort study of 261 type 1 diabetic adults with preserved kidney function at baseline, who were followed up for a mean period of 5.2 years, NAFLD (diagnosed by ultrasonography) was associated with an approximately threefold increased incidence of CKD. Figure 3 shows a Kaplan-Meier analysis of incidence curves for CKD over the follow-up period in patients with and without NAFLD at baseline. ${ }^{109}$ Notably, measurement of NAFLD improved risk prediction for $\mathrm{CKD}$ in this patient cohort, independently of traditional cardiorenal risk factors (age, sex, duration of diabetes, haemoglobin A1c, hypertension, baseline eGFR and microalbuminuria (ie, the last two factors being the strongest known risk factors for CKD)). ${ }^{109}$

A 2014 systematic review and meta-analysis of 33 observational studies (including 20 cross-sectional and 13 prospective studies and involving nearly 64000 individuals) examined the relationship between NAFLD and risk of CKD. ${ }^{110}$ NAFLD was diagnosed by biochemistry, imaging or histology, and CKD as either eGFR $<60 \mathrm{~mL} / \mathrm{min} / 1.73 \mathrm{~m}^{2}$ or proteinuria. The results of this meta-analysis showed that NAFLD was associated with a nearly twofold increase in the prevalence and incidence of CKD. Similarly, although only a few studies used liver biopsy to
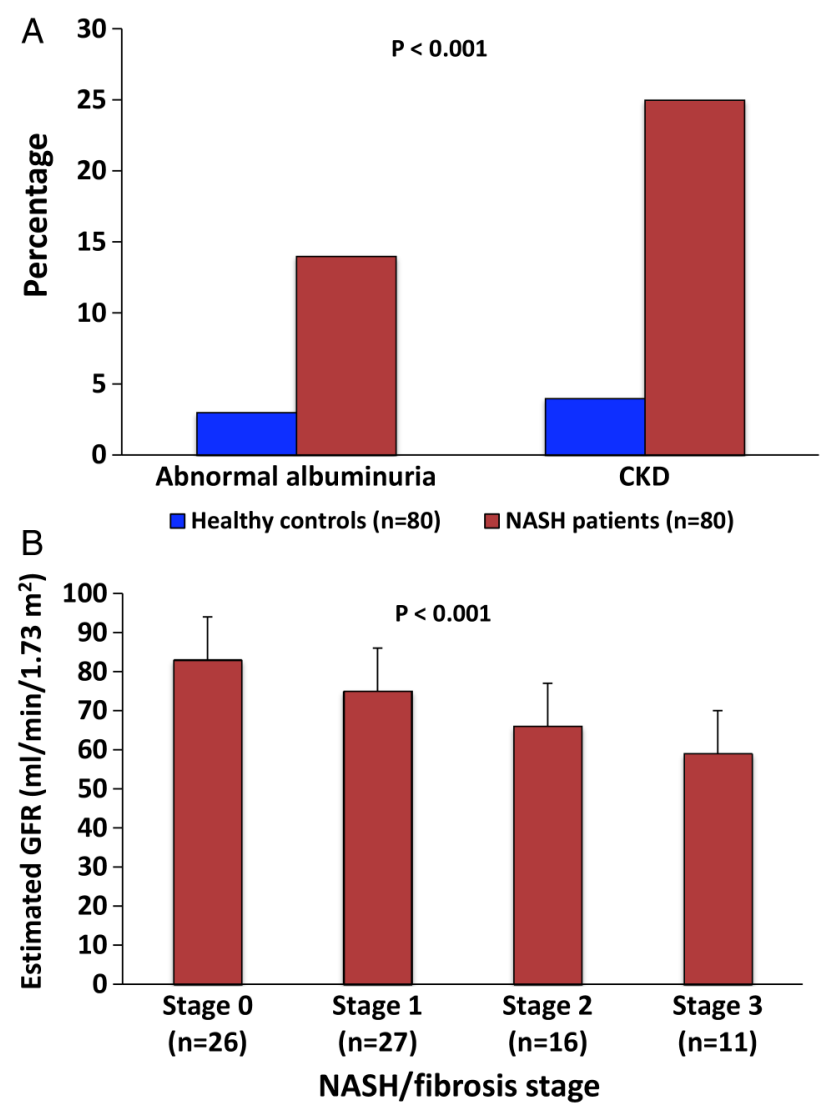

Figure 2 Kidney function in patients with non-alcoholic steatohepatitis (NASH). (A) Shows the prevalence of both abnormal albuminuria (ie, urinary albumin-to-creatinine ratio $\geq 30 \mathrm{mg} / \mathrm{g}$ ) and chronic kidney disease (CKD, defined as estimated glomerular filtration rate (eGFR), Modification of Diet in Renal Disease (MDRD) $<60 \mathrm{~mL} / \mathrm{min} /$ $1.73 \mathrm{~m}^{2}$ or abnormal albuminuria) in 80 overweight patients with biopsy-proven NASH and 80 non-steatotic control individuals who were matched for age, sex and body mass index. (B) Shows the adjusted means $( \pm$ SDs) of eGFR in patients with NASH according to the histological stage of hepatic fibrosis (from 0 , indicating no fibrosis, to 3 , indicating advanced fibrosis; patients with cirrhosis (ie, those with stage 4 fibrosis) were not included in the study). Data have been adjusted for age, sex, body mass index, waist circumference, hypertension status, plasma triglyceride concentrations and insulin resistance (as estimated on the basis of a homoeostasis model assessment) (adapted from Targher et al [99]).

diagnose NAFLD, the presence of histologically confirmed NASH was associated with an approximately 2.5 -fold increased prevalence and incidence of CKD than simple steatosis. Moreover, the presence of advanced hepatic fibrosis was associated with a remarkably greater prevalence and incidence of CKD than non-advanced fibrosis. In all of these analyses, the significant association between NAFLD and CKD persisted after adjustment for pre-existing diabetes, hypertension and other cardiorenal risk factors. ${ }^{110}$

In line with these observations, in a cohort of 261 patients with biopsy-proven NASH, who were treated with lifestyle modifications for 52 weeks, Vilar-Gomez et $a l^{111}$ found that improvement in histological NASH-related end points, achieved by weight loss, was independently associated with improvement in kidney function parameters.

Collectively, these findings provide robust evidence of a strong association between the presence and severity of NAFLD with the stage and risk of developing CKD. Therefore, these findings call for a more active and systematic search for CKD in 
Figure 3 Risk of incident chronic kidney disease (CKD) in patients with type 1 diabetes and non-alcoholic fatty liver disease (NAFLD). Incidence curves for CKD (defined as occurrence of estimated glomerular filtration rate (eGFR) $<60 \mathrm{~mL} / \mathrm{min} / 1.73 \mathrm{~m}^{2}$ or macroalbuminuria) in a cohort of 261 adults with type 1 diabetes with (dotted line) and without (solid line) NAFLD on ultrasonography over a mean follow-up period of 5.2 years. All patients had preserved kidney function $\left(e G F R_{M D R D} \geq 60 \mathrm{~mL} / \mathrm{min} / 1.73 \mathrm{~m}^{2}\right)$ and no macroalbuminuria at baseline.

Patients with known liver diseases and those with a prior history of cardiovascular disease were excluded from the study (adapted from Targher et al [109]).

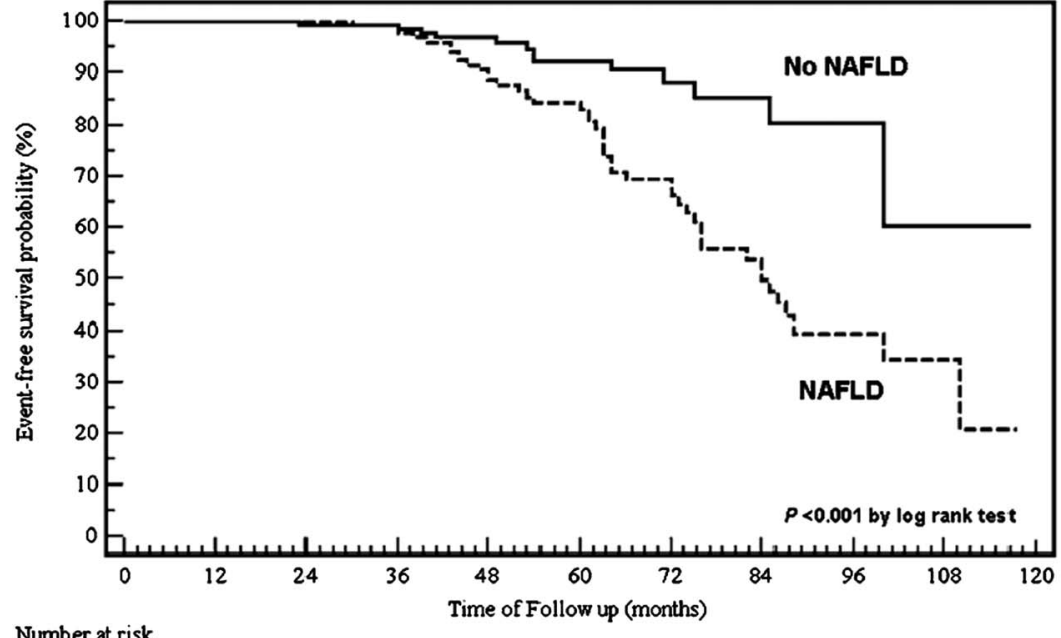

Number at risk

No NAFLD 130

130

129

120

$99 \quad 68$

$38 \quad 17$

11

1

0

NAFLD 131

131

11894

$68 \quad 41$ patients with NAFLD. However, it is important to underline that the quality of some of the published studies is limited, and that causality remains to be definitely proven in larger clinical trials with incident CKD outcomes that focus on treatments for liver disease in NAFLD. Notably, all these studies used creatinine-based equations to estimate GFR (which do not perform well in patients with cirrhosis or severe obesity) instead of direct GFR measurements to define CKD. Furthermore, no detailed information was available in these studies about specific renal pathology/morphology associated with NAFLD. Further research is also needed to better elucidate the underlying mechanisms by which NAFLD contributes to the development and progression of CKD.

\section{EPIDEMIOLOGICAL DATA LINKING NAFLD TO RISK OF TYPE 2 DIABETES}

Obesity and insulin resistance are key pathogenic factors for NAFLD and T2DM, and thus these two diseases commonly coexist; NAFLD is present in up to $75 \%$ of patients with T2DM, whereas the prevalence of T2DM in adults with NAFLD is approximately $10 \%-18 \% .^{112-114}$ In the absence of T2DM, NAFLD is a marker of insulin resistance independent of obesity or visceral adiposity, and predicts a greater deterioration in insulin sensitivity with weight gain compared with matched non-NAFLD individuals. ${ }^{115}$ Concomitant with these mechanistic observations, there is now robust evidence from observational studies demonstrating an increased risk of incident T2DM following a diagnosis of NAFLD. It is also notable that, in dual biopsy studies, the development of incident T2DM was the strongest predictor of progression to NASH and liver fibrosis. ${ }^{1}$

A significant association between mildly elevated serum liver enzymes and increased risk of incident T2DM was described nearly two decades ago. ${ }^{116}$ A meta-analysis of 20 observational studies (involving a total of 117000 subjects belonging to different ethnic groups) found a 1.6-2.0-fold increased risk of incident T2DM when comparing the highest versus the lowest quartiles of serum aminotransferase levels over a median follow-up of 5 years. ${ }^{117}$ Nevertheless, serum aminotransferase levels are relatively insensitive markers of NAFLD. Predictive non-invasive scores, such as the FLI or the NAFLD fatty liver score, incorporate serum liver enzymes with various metabolic variables, such as BMI and serum triglyceride levels, into their diagnostic algorithms, resulting in greater diagnostic accuracy for NAFLD than serum liver enzymes alone. Both of these noninvasive scores have been demonstrated to predict incident T2DM, however it is difficult to distinguish whether it is NAFLD itself or the metabolic variables within these scores, which are responsible for the increased T2DM risk. ${ }^{118}$

More robust evidence of the existence of an association between NAFLD and incident T2DM arises from multiple large cohort studies with a median follow-up period of at least 5 years that used ultrasonography or, less commonly, CT to diagnose NAFLD (table 2). After adjusting for several potential confounding factors, NAFLD has been associated with a 1.52-fold increased risk of new-onset T2DM over a 5-10-year follow-up period. ${ }^{119-129}$ It is notable that the increased T2DM risk associated with NAFLD was observed in both sexes; however it was typically higher in men, mirroring the male predominance in T2DM. ${ }^{124} 128129$

Notably, the risk of new-onset T2DM appears to diminish over time following the improvement or resolution of NAFLD, with some Asian cohort studies demonstrating T2DM incidence returning to that of subjects without fatty liver on ultrasonography. ${ }^{129-131}$ This is likely to be closely related to temporal changes in body weight, although one study found NAFLD resolution to be associated with a lower incidence of T2DM independently of change in BMI. ${ }^{130}$ Another study found NAFLD improvement was only associated with a reduced risk of incident T2DM in those who had a concomitant BMI reduction over time. ${ }^{129}$

NAFLD appears to be additive to established metabolic risk factors of obesity and insulin resistance in increasing the risk of incident T2DM. ${ }^{120127128132}$ One cohort study of 12853 South Korean non-diabetic individuals found that the risk of incident T2DM over 5 years increased 2.7-fold with a baseline diagnosis of fatty liver on ultrasound, and 3.7-fold with insulin resistance determined by the homoeostasis model of assessment, whereas their combination increased the risk to 6.7 -fold. ${ }^{132}$ Nevertheless, the interaction between insulin resistance, metabolic syndrome and NAFLD is complex with several studies suggesting the association between NAFLD and incident T2DM to be modified by the severity of underlying insulin resistance or baseline body weight; two studies have also found that the risk of incident 


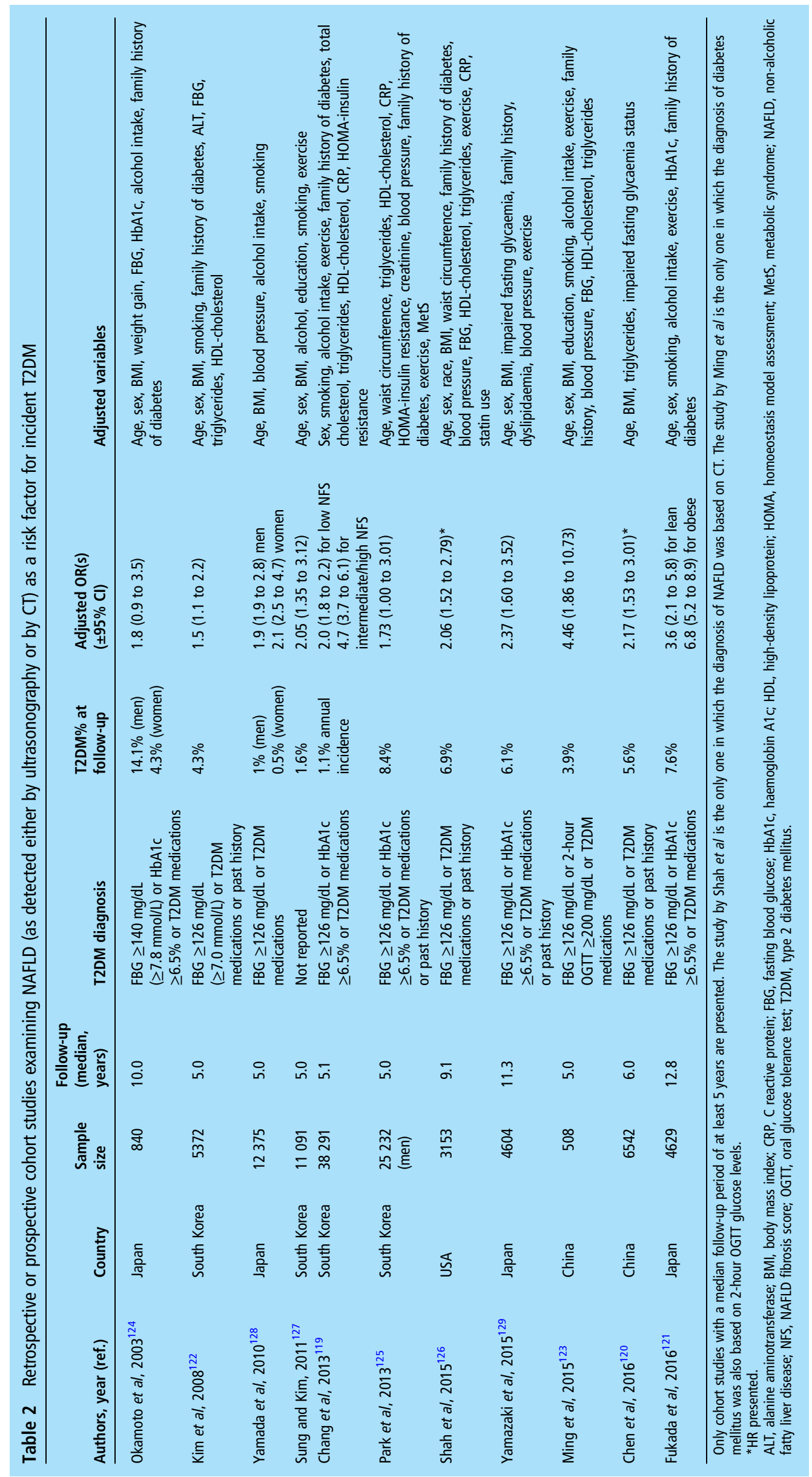


T2DM with NAFLD is limited to patients with either baseline-impaired fasting glycaemia or those with high baseline levels of insulin resistance. ${ }^{127} 133$ Similarly, a Finnish study of hypertensive patients followed for 21 years found that NAFLD was associated with an increased risk of incident T2DM when coexisting with the metabolic syndrome, but not in its absence. ${ }^{134}$ Surprisingly, however, two Japanese studies found the magnitude of risk of incident T2DM to be higher in patients who have NAFLD but are lean or have lower BMI values. ${ }^{121} 128$ This finding might be, at least in part, due to the lower sensitivity of ultrasonography for detecting NAFLD with increasing BMI, potentially leading to some misclassification bias.

The severity of underlying NAFLD appears to influence the magnitude of future risk of T2DM, with increasing risk that parallels the severity of hepatic steatosis on ultrasonography or CT. ${ }^{122} 125126$ Importantly, in these studies the T2DM risk associated with NAFLD remained significant even after adjustment for a range of common T2DM risk factors. Similarly, a Korean study found that only patients with NAFLD with elevated alanine-aminotransferase levels had an increased risk of incident T2DM, which may reflect more severe hepatic steatosis or the histologically more aggressive subtype of NASH. ${ }^{135}$ Correspondingly, patients with NASH have a higher risk of developing T2DM compared with those with simple steatosis, although it is not clear whether this risk is independent of confounding factors such as obesity. ${ }^{50}$ Lastly, after stratification of patients with NAFLD according to likelihood of advanced hepatic fibrosis by the NFS, those with high or intermediate NFS values are more than twice as likely to develop T2DM than those with low NFS values. ${ }^{119}$ Although there is now convincing evidence that NAFLD is strongly associated with the risk of incident CKD in patients with type 1 or type 2 diabetes (as discussed previously), it remains unclear whether NAFLD or NASH are also independent risk factors for diabetic retinopathy (another microvascular complication of diabetes), with evidence limited only to cross-sectional studies with conflicting conclusions. $^{89} 136$

It is important to underline that the majority of observational studies that examined the relationship between NAFLD and risk of incident T2DM are retrospective and originate from Asia where large populations undergo regular health check-ups including liver ultrasonography. Only one study from the USA found CT-determined hepatic steatosis to be independently associated with increased risk of incident T2DM in a population that included Caucasians, Blacks and Hispanics; however whether this risk differs according to race is unknown as a stratified analysis was not performed. ${ }^{126}$ Additionally, as Asian and non-Asian populations have different adipose tissue distributions and cultural/genetic backgrounds, further evidence is needed in different ethnic and racial groups. Finally, the study by Ming et $a l^{123}$ was the only one in which the diagnosis of diabetes was also based on 2-hour postload plasma glucose levels.

Currently, it is unclear whether NAFLD is causally related to T2DM development or is simply a marker of other shared risk factors, such as visceral adipose tissue. Notably, some genetic conditions leading to intrahepatic triglyceride accumulation, such as familial hypobetalipoproteinaemia, do not lead to increased insulin resistance, and genetic polymorphisms in the PNPLA3 gene (that also correlate with higher intrahepatic triglyceride content and increased risk of NASH, but are not systematically associated with insulin resistance and metabolic syndrome features) only appear to associate with T2DM in the presence of obesity, suggesting that other factors in addition to hepatic steatosis are important for the development of T2DM. ${ }^{69} 137138$
Despite the above caveats, a large number of cohort studies clearly demonstrated NAFLD to be associated with an approximate doubling of risk of incident T2DM. This association appears to be dose-dependent and is ameliorated with NAFLD improvement or resolution over time. Consequently, current clinical guidelines recommend routine screening of patients with NAFLD for T2DM with fasting blood glucose or haemoglobin A1c levels or with a $75 \mathrm{~g}$ oral glucose tolerance test in high-risk groups. $^{139}$

\section{EPIDEMIOLOGICAL DATA LINKING NAFLD TO RISK OF COLORECTAL ADENOMAS/CANCER AND OTHER EXTRAHEPATIC NEOPLASMS}

Patients with NAFLD typically have a range of metabolic risk factors associated with the development of neoplasia, including T2DM and obesity; consequently, extrahepatic malignancy is the second most common cause of death among patients with NAFLD. ${ }^{4} 5$ In addition, one community-based cohort study of patients with T2DM suggested that NAFLD was associated with an increased risk of death from malignancy, suggesting an independent effect of NAFLD over and above coexisting metabolic risk factors. ${ }^{140}$

The strongest association between NAFLD and extrahepatic neoplasia has been reported with colorectal adenomas (as summarised in table 3). One Taiwanese study of 1522 individuals found a diagnosis of NAFLD in subjects with a normal baseline colonoscopy was associated with a $45 \%$ increased risk of future adenoma on subsequent colonoscopy 5 years later. ${ }^{141}$ This association remained significant after adjustment for age, sex, smoking and metabolic risk factors. Two subsequent meta-analyses of five observational studies each, demonstrated a 1.5-1.7-fold increased risk of colorectal adenomas. ${ }^{142} 143$ NAFLD was also associated with multiple colorectal adenomas $(\geq 3)$ and a tendency towards proximal lesions (right-sided colonic adenomas). Whether the risk of colorectal adenoma increases with NASH compared with simple steatosis is unclear with two relatively small cross-sectional studies demonstrating conflicting results. ${ }^{144} 145$ Recently, a large cross-sectional study of 26540 asymptomatic individuals from South Korea however, found the risk for any colorectal neoplasia and advanced colorectal neoplasia to be particularly increased in patients with NAFLD with high NFS or other non-invasive fibrosis scores. ${ }^{146}$

The risk of developing colorectal cancer also appears to be increased, with two large cohort studies of Korean and Chinese individuals undergoing colonoscopy $(n=2315$ and $n=5517$, respectively) finding a diagnosis of NAFLD on ultrasound to be significantly associated with an increased cancer risk of 1.87 (95\% CI 1.4 to 2.6) and 3.08 (95\% CI 1.02 to 9.3), respectively. ${ }^{147} 148$ Preliminary reports have also linked NAFLD with the occurrence of gastric and prostate cancers ${ }^{149}$, however further data are required before any definitive conclusions can be made. Further research is also needed to establish whether risk of these extrahepatic cancers differs with severity of NAFLD.

Nevertheless, the studies to date have been predominately cross-sectional, limiting any inference about causality and largely restricted to Asian populations. Currently, although a diagnosis of NAFLD is not sufficient to recommend screening colonoscopy, evaluation of colonic symptoms and ensuring patients are enrolled in colorectal cancer screening programmes as per recommendations for the general population is strongly recommended. Further evidence is needed to clarify the risk in patients aged 40-50 years who are not currently within routine screening guidelines. However, if the potential adverse impact of NAFLD on risk of colorectal adenomas/cancer will be 


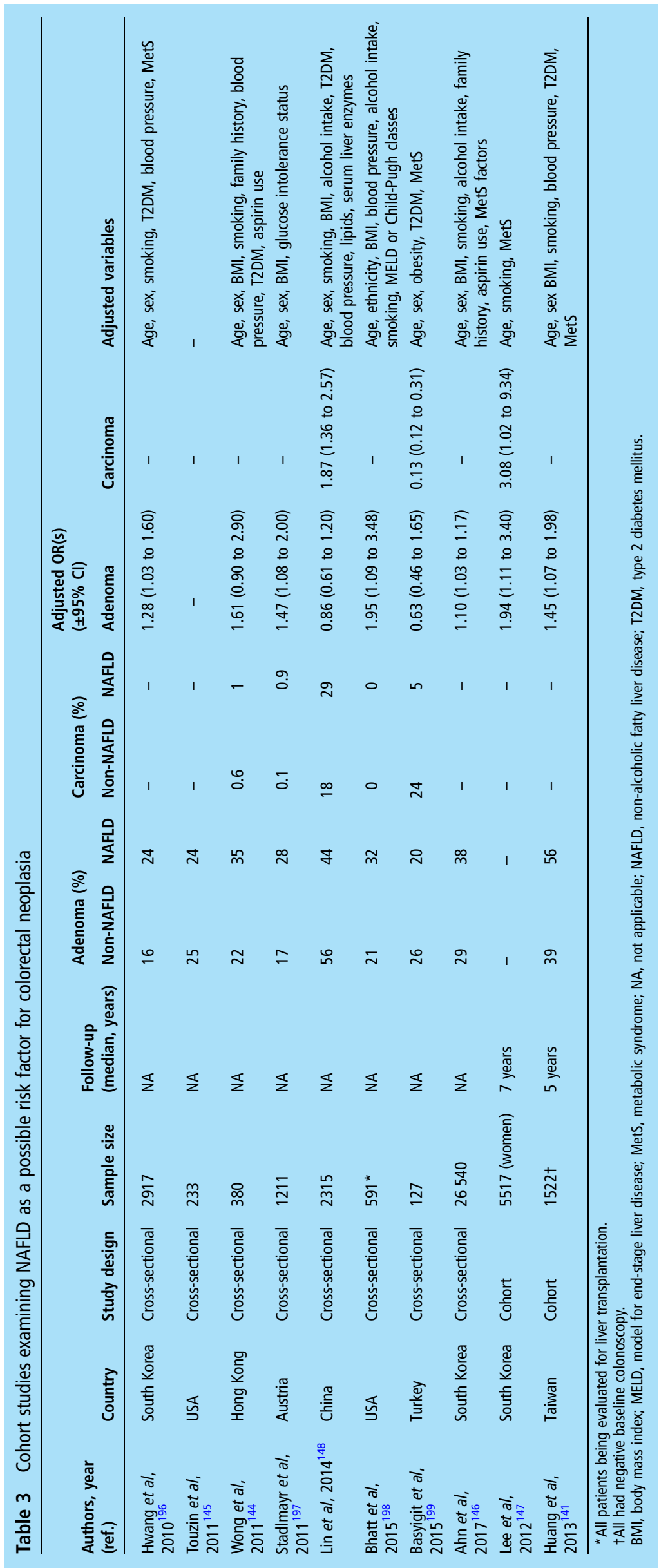


confirmed in future large-scale prospective studies, there may be significant implications for screening and surveillance strategies given the growing number of patients with NAFLD.

\section{EPIDEMIOLOGICAL DATA LINKING NAFLD TO RISK OF OSTEOPOROSIS}

NAFLD is associated with multiple factors linked to decreased bone mineral density (BMD), including T2DM, obesity, insulin resistance, chronic inflammation, vitamin $\mathrm{D}_{3}$ deficiency and low levels of physical activity. ${ }^{150}$ Consequently, several cross-sectional and case-control studies involving both adults and adolescents have found a significant association between low BMD or osteoporosis and NAFLD ${ }^{150}$. Worryingly, this association has been also reported in the paediatric age group, with one study of 38 obese children with biopsy-proven NAFLD reporting significantly lower BMD compared with 38 non-steatotic children matched for age, sex, ethnicity and BMI. ${ }^{151}$ The degree of BMD fell with increasing severity of liver disease with patients with NASH having a lower level than those with simple steatosis.

In adults, the association between NAFLD and osteoporosis is less consistent, with a meta-analysis of a total of 1276 individuals finding no significant differences in BMD between subjects with or without NAFLD, whereas a significant association with BMI was found. ${ }^{152}$ Significant heterogeneity between studies however, limits conclusions from this meta-analysis. Subsequently, three large cohort studies totalling over 2500 individuals, all found a significant inverse association between BMD and NAFLD, although interestingly the association was limited to men and not seen in postmenopausal women. ${ }^{153-155}$ The association between NAFLD and BMD was modest, however, and remained significant in these studies even after adjusting for several potential confounding factors, including age, weight, alcohol intake, smoking and metabolic risk factors. Increasing ultrasonographic severity of hepatic steatosis as well as serum alanine-aminotransferase levels correlated inversely with BMD. ${ }^{153}$ A further cohort study of 7797 Chinese adults aged $>40$ years confirms the existence of a sexspecific association with a higher rate of recent osteoporotic fractures in men with NAFLD compared with those without $3.6 \%$ vs $1.7 \%, \mathrm{p}=0.003)$, with no difference seen in women. ${ }^{156}$ Overall, men with NAFLD had a 2.5 -fold increased risk of recent osteoporotic fractures even after adjustment for age, smoking, alcohol intake, physical activity, metabolic risk factors, kidney function and use of glucocorticoids or osteoporosis medications. ${ }^{156}$ The male-specific association suggests a sex-specific interaction between hepatic steatosis and bone metabolism, or that other factors in women, such as oestrogen status, may dominate the risk of osteoporosis.

Collectively, these findings argue for awareness of BMD among patients with NAFLD. However, it is important to note that the available data are limited to cross-sectional studies largely from Asian populations. The majority of studies focus on BMD rather than bone fracture rate and further evidence is required to clarify whether patients with NAFLD (in particular men) should be screened for osteoporosis.

\section{PUTATIVE MECHANISMS LINKING NAFLD TO EXTRAHEPATIC CONDITIONS}

Extrahepatic pathological conditions in NAFLD are closely linked to the presence of local (ie, in the liver, adipose tissue, vessels and heart) and systemic inflammation. Chronic low-grade inflammation accompanies many metabolic disorders, such as T2DM or NAFLD. ${ }^{157}$ It has been demonstrated that inflammatory pathways such as IL-1-type cytokines are driving forces of disease processes in NAFLD and correlate with prognosis of liver disease. ${ }^{158} 159$ Hepatic inflammation might be responsible for the overall degree of hepatic fibrosis and thereby prognosis of this disorder and might also control insulin resistance. Hepatic/peripheral insulin resistance (ie, a hallmark in most patients with NAFLD) and metabolic inflammation are frequently observed in parallel, and research from the past has tried to connect these two phenomena. ${ }^{160}$ The origin of chronic inflammatory processes observed in NAFLD is still a matter of discussion as multiple parallel 'hits' might control and regulate disease process. ${ }^{161}$ Besides lipotoxicity, the GI tract with its significantly altered microbiota could reflect one of the early events in disease development. In addition, abdominal visceral adipose tissue accumulation is another 'hot' candidate reflecting a major site of chronic inflammation in NAFLD as adipose tissue inflammation is commonly observed in obesity, T2DM and NAFLD. Indeed, it has been shown that the inflamed ('dysfunctional') adipose tissue in obesity might generate almost $50 \%$ of circulating IL-6 thereby contributing substantially to systemic chronic inflammation. ${ }^{162}$ The importance of visceral adipose tissue as site of chronic inflammation is further supported by some studies demonstrating that the lack of adipocyte $5^{\prime}$ AMP-activated protein kinase worsens insulin resistance and liver disease by affecting brown and beige adipose tissue function. ${ }^{163}$ Additionally, adipose tissue-specific insulin receptor knockout mice develop more severe NAFLD with histological evidence of ballooning degeneration further supporting the notion that the adipose tissue is of key importance in the development and progression of NAFLD. ${ }^{164}$ In contrast, recent research has found that adipose tissue type I interferon signalling may protect from metabolic dysfunction. ${ }^{165}$

\section{Nutrition and dietary factors}

Nutrition is critically involved in the pathogenesis of NAFLD, besides the fact that NAFLD is mainly a disease of the obese population. Overnutrition and certain dietary factors have been demonstrated to induce chronic low-grade inflammation. ${ }^{166} 167$ Epidemiological studies have shown that diet can affect inflammatory processes and the immune system directly, and also through interactions with the gut microbiota. Western diets are able to promote chronic inflammatory processes by many pathways. A high salt content may induce IL-17 producing T helper cells and thereby inflammation. ${ }^{168}$ Dietary fatty acids promote inflammation through several mechanisms, including direct effects on immune cells, activation of toll-like receptors and cytokine cascades, ${ }^{169}$ and affect intestinal permeability. ${ }^{170}$ Mice with a loss of junctional adhesion molecule A (JAM-A) have a defective intestinal epithelial barrier and exhibit more prominent NASH, and colonic tissue from patients with NAFLD have lower levels of JAM-A and higher levels of inflammation compared with healthy controls. ${ }^{171}$ In healthy individuals, a high-fat Western diet induces endotoxaemia and, therefore, might contribute to the observed chronic inflammation in NAFLD. ${ }^{172} \mathrm{~A}$ diet rich in saturated milk-derived fatty acids worsens colitis in IL-10 deficient mice via profound changes of the gut microbiota and an increased Th1 cell response. ${ }^{173}$ Many studies have also associated dietary phosphatidylcholine and L-carnitine consumption with generation of specific metabolites (ie, trimethylamine (TMA) and TMA N-oxide) and future CVD events. ${ }^{174}$ Not surprisingly, dietary interventions might be able to reverse a Western-diet induced liver phenotype as shown, for example, for citrulline. ${ }^{175}$ Overall, the composition of Western diets might contribute to the development/progression of NAFLD via generation of overweight and obesity, and induce activation of specific inflammatory pathways. 


\section{Gut microbiota}

Evidence is increasing that the gut microbiota might contribute to the pathogenesis of NAFLD. NAFLD reflects a prototypical disease of innate immunity and it is expected that certain bacteria in the intestinal tract activate various pathways of innate immunity in the case of disease. ${ }^{176}$ In several mouse models, the microbiota affects hepatic steatosis and fat storage. Human studies from the past years have also supported a role for the microbiota in NAFLD. Actinobacteria were increased whereas Bacteroidetes were reduced dependent on liver disease activity as demonstrated in a paediatric study population. ${ }^{177}$ At species levels, Oscillobacter decreased in NAFLD whereas Ruminococcus, Blautia and Dorea increased especially in patients with NASH. ${ }^{177}$ Another study assessed the gut microbiota and severity of histology-proven NAFLD in 57 patients. ${ }^{178}$ Bacteroides abundance increased depending on severity of disease, whereas Prevotella abundance decreased. Ruminococcus abundance increased in more severe disease, especially if advanced hepatic fibrosis was diagnosed. ${ }^{178}$ Overall, many studies from the past years clearly highlighted a role for the gut microbiota in obesity-related disorders supporting the assumption that NASH might be associated with a 'microbiome signature' which could contribute to initiation of inflammation. It has to be acknowledged, however, that these early studies are rather descriptive and neither studies (either preclinical or clinical) have so far identified certain bacterial strains, which act proinflammatory and are indeed involved in disease pathogenesis. The altered microbiome in patients with NAFLD could also be involved in the development of NAFLD-associated malignancy both in the liver ${ }^{179}$ and in the GI tract. ${ }^{180}$

\section{Cytokines/adipocytokines}

It is well established that proinflammatory cytokines and transcription factors are highly expressed in various tissues, such as the adipose tissue or liver, in NAFLD and a large number of different immune cells and, especially, tissue macrophages may contribute to the inflammatory phenotype. Various proinflammatory cytokines, such as TNF- $\alpha$ or IL-6, are activated in different tissues but especially in adipose tissue and in the liver of patients with NAFLD/NASH. ${ }^{181}$ There is substantial evidence that activation of the transcription of nuclear factor- $\mathrm{B}(\mathrm{NF}-\kappa \mathrm{B})$ and downstream inflammatory signalling pathways are involved in metabolic liver inflammation and associated hepatic insulin resistance. ${ }^{182} 183$ Receptor activator of NF- $\mathrm{KB}$, a prototypical activator of NF- $\mathrm{B}$, regulates hepatic insulin sensitivity. ${ }^{184}$ Deletion of hepatic MyD88 results in liver inflammation and hepatic insulin resistance also affecting the gut microbiota composition and metabolome. ${ }^{185}$ Therefore, several studies using hepatocytespecific targeting of genes have now strongly supported the notion that hepatocytes are major metabolic 'players' in the development of metabolic inflammation in and beyond the liver.

Chronic inflammation and cytokine activation is a driving force in the evolution of malignancy and, therefore, it is expected that patients with NAFLD exhibit a high rate of liver and extrahepatic malignancies. ${ }^{144} 186$ Chronic IL-6 overexpression has been found to result in the generation of liver tumours. ${ }^{187}$ In these studies, the development of obesitypromoted hepatocellular carcinoma was dependent on enhanced production of the tumour-promoting cytokines IL-6 and TNF- $\alpha$, which cause hepatic inflammation and activation of the oncogenic transcription factor STAT3. Besides several cytokines certain adipocytokines, such as adiponectin and leptin, might contribute to systemic inflammatory processes and associated pathologies in NAFLD. ${ }^{188} 189$ For example, NAFLD is associated with hypoadiponectinaemia and lower adiponectin levels correlate with colorectal cancer/adenoma incidence. ${ }^{190} 191$ Low-level plasma adiponectin is especially associated with KRAS-mutant colorectal cancer risk. ${ }^{192}$ Therefore, it may be concluded that chronic activation of cytokine/adipocytokine cascades as observed in NAFLD might contribute substantially to the extrahepatic chronic diseases observed in NAFLD ranging from CVD to osteoporosis and especially also hepatic and extrahepatic cancers.

\section{CONCLUSIONS}

This review further reinforces the view that NAFLD is a multisystem disease that affects many extrahepatic organ systems and interacts with the regulation of multiple metabolic pathways. NAFLD is associated with liver-related morbidity and mortality, and with an increased risk of developing important extrahepatic chronic diseases, such as CVD, T2DM and CKD. Emerging data also suggest NAFLD may be a risk factor for colonic adenomas/cancer and decreased BMD, particularly among men. Clear evidence indicates that CVD is the leading cause of death in patients with NAFLD. This implies that patients with NAFLD should undergo careful cardiovascular surveillance. In line with this implication, given that CVD complications dictate the outcome(s) in patients with NAFLD more frequently and to a greater extent than does the progression of liver disease, the recent European clinical practice guidelines have recommended CVD risk assessment in all patients with NAFLD. ${ }^{139}$

There is a growing body of epidemiological and experimental evidence suggesting that NAFLD, especially its necroinflammatory form, exacerbates hepatic/peripheral insulin resistance, predisposes to atherogenic dyslipidaemia and releases a variety of proinflammatory, procoagulant, thrombogenic and profibrogenic factors that may promote the development of CVD, CKD, T2DM and other extrahepatic chronic diseases.

However, although all these mechanisms plausibly link NAFLD to the development and progression of CVD, CKD and other extrahepatic diseases, no studies to date have proven a cause-and-effect relationship and further research is certainly needed to gain mechanistic insights into the pathophysiology linking NAFLD to the development and progression of these extrahepatic chronic diseases.

In the meantime, we believe that the clinical implication of these findings is that a diagnosis of NAFLD may identify a subset of the general population, which is exposed to an increased risk of developing some important extrahepatic chronic diseases. Therefore, patients with NAFLD might benefit from more intensive surveillance or early treatment interventions to decrease the risk of developing CVD, CKD, T2DM and other extrahepatic manifestations. Thus, clinicians who manage patients with NAFLD, especially those with NASH with varying amounts of fibrosis, should focus on liver disease, and should recognise the increased risk of CVD, CKD, T2DM and other serious extrahepatic manifestations of these patients, screen them for conventional risk factors and undertake early, aggressive risk factor modification(s).

Funding QMA is a member of the EPOS (Elucidating Pathways of Steatohepatitis) consortium funded by the Horizon 2020 Framework Program of the European Union under Grant Agreement 634413. HT is supported by the excellence initiative (Competence Centers for Excellent Technologies-COMET) of the Austrian Research Promotion Agency FFG: Research Center of Excellence in Vascular Ageing Tyrol, VASCage (K-Project Nr. 843536) funded by the BMVIT, BMWFW, the

Wirtschaftsagentur Wien and the Standortagentur Tirol. GT is supported in part by grants from the University School of Medicine of Verona, Verona, Italy. 
Competing interests None declared.

Provenance and peer review Commissioned; externally peer reviewed.

\section{REFERENCES}

1 McPherson S, Hardy $T$, Henderson $E$, et al. Evidence of NAFLD progression from steatosis to fibrosing-steatohepatitis using paired biopsies: Implications for prognosis and clinical management. J Hepatol 2015;62:1148-55.

2 Singh $\mathrm{S}$, Allen AM, Wang Z, et al. Fibrosis progression in nonalcoholic fatty liver vs nonalcoholic steatohepatitis: a systematic review and meta-analysis of paired-biopsy studies. Clin Gastroenterol Hepatol 2015;13:643-54.e9.

3 Anstee QM, Targher G, Day CP. Progression of NAFLD to diabetes mellitus, cardiovascular disease or cirrhosis. Nat Rev Gastroenterol Hepatol 2013; 10:330-44.

4 Ekstedt $\mathrm{M}$, Hagström $\mathrm{H}$, Nasr $\mathrm{P}$, et al. Fibrosis stage is the strongest predictor for disease-specific mortality in NAFLD after up to 33 years of follow-up. Hepatology 2015:61:1547-54.

5 Angulo $P$, Kleiner DE, Dam-Larsen $S$, et al. Liver fibrosis, but no other histologic features, is associated with long-term outcomes of patients with nonalcoholic fatty liver disease. Gastroenterology 2015;149:389-97.e10.

6 Angulo P. Nonalcoholic fatty liver disease. N Engl J Med 2002;346:1221-31.

7 Marchesini G, Bugianesi E, Forlani G, et al. Nonalcoholic fatty liver, steatohepatitis, and the metabolic syndrome. Hepatology 2003;37:917-23.

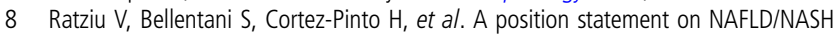
based on the EASL 2009 special conference. J Hepatol 2010;53:372-84.

9 Kotronen A, Westerbacka J, Bergholm R, et al. Liver fat in the metabolic syndrome. J Clin Endocrinol Metab 2007;92:3490-7.

10 Li Y, Xu C, Yu C, et al. Association of serum uric acid level with non-alcoholic fatty liver disease: a cross-sectional study. J Hepatol 2009;50:1029-34.

11 Sirota JC, McFann K, Targher G, et al. Elevated serum uric acid levels are associated with non-alcoholic fatty liver disease independently of metabolic syndrome features in the United States: liver ultrasound data from the National Health and Nutrition Examination Survey. Metab Clin Exp 2013;62:392-9.

12 Petta S, Cammà C, Cabibi D, et al. Hyperuricemia is associated with histological liver damage in patients with non-alcoholic fatty liver disease. Aliment Pharmacol Ther 2011;34:757-66.

13 Hui JM, Hodge A, Farrell GC, et al. Beyond insulin resistance in NASH: TNF-alpha or adiponectin? Hepatology 2004;40:46-54.

14 Targher $\mathrm{G}$, Bertolini L, Rodella S, et al. Associations between plasma adiponectin concentrations and liver histology in patients with nonalcoholic fatty liver disease. Clin Endocrinol (Oxf) 2006:64:679-83.

15 Bugianesi E, Pagotto U, Manini R, et al. Plasma adiponectin in nonalcoholic fatty liver is related to hepatic insulin resistance and hepatic fat content, not to liver disease severity. J Clin Endocrinol Metab 2005;90:3498-504.

16 Targher G, Bertolini L, Scala L, et al. Associations between serum 25-hydroxyvitamin D3 concentrations and liver histology in patients with non-alcoholic fatty liver disease. Nutr Metab Cardiovasc Dis 2007;17:517-24

17 Targher G, Chonchol M, Zoppini G, et al. Risk of chronic kidney disease in patients with non-alcoholic fatty liver disease: is there a link? J Hepatol 2011;54:1020-9.

18 Targher G, Chonchol M, Miele L, et al. Nonalcoholic fatty liver disease as a contributor to hypercoagulation and thrombophilia in the metabolic syndrome. Semin Thromb Hemost 2009;35:277-87.

19 Sookoian S, Castaño GO, Burgueño AL, et al. Circulating levels and hepatic expression of molecular mediators of atherosclerosis in nonalcoholic fatty liver disease. Atherosclerosis 2010;209:585-91.

20 Wieckowska A, Papouchado BG, Li Z, et al. Increased hepatic and circulating interleukin-6 levels in human nonalcoholic steatohepatitis. Am J Gastroenterol 2008;103:1372-9.

21 Sookoian S, Gianotti TF, Rosselli MS, et al. Liver transcriptional profile of atherosclerosis-related genes in human nonalcoholic fatty liver disease. Atherosclerosis 2011;218:378-85.

22 Weston CJ, Shepherd EL, Claridge LC, et al. Vascular adhesion protein-1 promotes liver inflammation and drives hepatic fibrosis. J Clin Invest 2015;125:501-20.

23 Vanni E, Marengo A, Mezzabotta L, et al. Systemic complications of nonalcoholic fatty liver disease: when the liver is not an innocent bystander. Semin Liver Dis 2015:35:236-49.

24 Targher G, Bertolini L, Padovani R, et al. Relation of nonalcoholic hepatic steatosis to early carotid atherosclerosis in healthy men: role of visceral fat accumulation. Diabetes Care 2004;27:2498-500.

25 Brea A, Mosquera D, Martín E, et al. Nonalcoholic fatty liver disease is associated with carotid atherosclerosis: a case-control study. Arterioscler Thromb Vasc Biol 2005;25:1045-50.

26 Volzke $H$, Robinson DM, Kleine $\mathrm{V}$, et al. Hepatic steatosis is associated with an increased risk of carotid atherosclerosis. World J Gastroenterol 2005;11:1848-53.

27 Fracanzani AL, Burdick L, Raselli S, et al. Carotid artery intima-media thickness in nonalcoholic fatty liver disease. Am J Med 2008;121:72-8.
28 Kozakova M, Palombo C, Eng MP, et al. Fatty liver index, gamma-glutamyltransferase, and early carotid plaques. Hepatology 2012;55:1406-15.

29 Salvi $\mathrm{P}$, Ruffini $\mathrm{R}$, Agnoletti $\mathrm{D}$, et al. Increased arterial stiffness in nonalcoholic fatty liver disease: the Cardio-GOOSE study. J Hypertens 2010;28:1699-707.

30 Lee YJ, Shim JY, Moon BS, et al. The relationship between arterial stiffness and nonalcoholic fatty liver disease. Dig Dis Sci 2012;57:196-203.

31 Villanova N, Moscatiello S, Ramilli S, et al. Endothelial dysfunction and cardiovascular risk profile in nonalcoholic fatty liver disease. Hepatology 2005:42:473-80.

32 Pacifico L, Anania C, Martino F, et al. Functional and morphological vascular changes in pediatric nonalcoholic fatty liver disease. Hepatology 2010:52:1643-51.

33 Sookoian S, Pirola CJ. Non-alcoholic fatty liver disease is strongly associated with carotid atherosclerosis: a systematic review. J Hepatol 2008;49:600-7.

34 Oni ET, Agatston AS, Blaha MJ, et al. A systematic review: burden and severity of subclinical cardiovascular disease among those with nonalcoholic fatty liver; should we care? Atherosclerosis 2013;230:258-67.

35 Sung KC, Wild SH, Kwag HJ, et al. Fatty liver, insulin resistance, and features of metabolic syndrome: relationships with coronary artery calcium in 10,153 people. Diabetes Care 2012:35:2359-64.

36 Goland S, Shimoni S, Zornitzki T, et al. Cardiac abnormalities as a new manifestation of nonalcoholic fatty liver disease: echocardiographic and tissue Doppler imaging assessment. J Clin Gastroenterol 2006:40:949-55.

37 Fallo F, Dalla Pozza A, Sonino N, et al. Non-alcoholic fatty liver disease is associated with left ventricular diastolic dysfunction in essential hypertension. Nutr Metab Cardiovasc Dis 2009;19:646-53.

38 Bonapace S, Perseghin G, Molon G, et al. Nonalcoholic fatty liver disease is associated with left ventricular diastolic dysfunction in patients with type 2 diabetes. Diabetes Care 2012;35:389-95.

39 Hallsworth K, Hollingsworth KG, Thoma C, et al. Cardiac structure and function are altered in adults with non-alcoholic fatty liver disease. J Hepatol 2013:58:757-62.

40 Perseghin G, Lattuada G, De Cobelli F, et al. Increased mediastinal fat and impaired left ventricular energy metabolism in young men with newly found fatty liver. Hepatology 2008;47:51-8.

41 Rijzewijk LJ, Jonker JT, van der Meer RW, et al. Effects of hepatic triglyceride content on myocardial metabolism in type 2 diabetes. J Am Coll Cardiol 2010;56:225-33.

42 Lautamäki R, Borra R, lozzo P, et al. Liver steatosis coexists with myocardial insulin resistance and coronary dysfunction in patients with type 2 diabetes. Am J Physiol Endocrinol Metab 2006;291:E282-90.

43 Stepanova M, Younossi ZM. Independent association between nonalcoholic fatty liver disease and cardiovascular disease in the US population. Clin Gastroenterol Hepatol 2012;10:646-50

44 Lazo M, Hernaez R, Bonekamp S, et al. Non-alcoholic fatty liver disease and mortality among US adults: prospective cohort study. BMJ 2011;343:d6891.

45 Targher G, Bertolini L, Padovani $R$, et al. Prevalence of nonalcoholic fatty liver disease and its association with cardiovascular disease among type 2 diabetic patients. Diabetes Care 2007;30:1212-18.

46 Mirbagheri SA, Rashidi A, Abdi S, et al. Liver: an alarm for the heart? Liver Int 2007:27:891-4

47 Assy N, Djibre A, Farah R, et al. Presence of coronary plaques in patients with nonalcoholic fatty liver disease. Radiology 2010;254:393-400.

48 Wong VW, Wong GL, Yeung JC, et al. Long-term clinical outcomes after fatty liver screening in patients undergoing coronary angiogram: a prospective cohort study. Hepatology 2016;63:754-63.

49 Wu S, Wu F, Ding Y, et al. Association of non-alcoholic fatty liver disease with major adverse cardiovascular events: a systematic review and meta-analysis. Sci Rep 2016;6:33386

50 Ekstedt M, Franzén LE, Mathiesen UL, et al. Long-term follow-up of patients with NAFLD and elevated liver enzymes. Hepatology 2006;44:865-73.

51 Söderberg C, Stål P, Askling J, et al. Decreased survival of subjects with elevated liver function tests during a 28-year follow-up. Hepatology 2010;51:595-602.

52 Musso G, Gambino R, Cassader M, et al. Meta-analysis: natural history of non-alcoholic fatty liver disease (NAFLD) and diagnostic accuracy of non-invasive tests for liver disease severity. Ann Med 2011;43:617-49.

53 Sinn DH, Cho SJ, Gu S, et al. Persistent nonalcoholic fatty liver disease increases risk for carotid atherosclerosis. Gastroenterology 2016;151:481-8.e1.

54 Sinn DH, Kang D, Chang Y, et al. Non-alcoholic fatty liver disease and progression of coronary artery calcium score: a retrospective cohort study. Gut 2017;66:323-9.

55 Pais R, Giral P, Khan JF, et al. Fatty liver is an independent predictor of early carotid atherosclerosis. J Hepatol 2016;65:95-102.

56 Ma J, Hwang SJ, Pedley A, et al. Bi-directional analysis between fatty liver and cardiovascular disease risk factors. J Hepatol 2017;66:390-7.

57 Kim D, Kim WR, Kim HJ, et al. Association between noninvasive fibrosis markers and mortality among adults with nonalcoholic fatty liver disease in the United States. Hepatology 2013;57:1357-65. 
58 Haring R, Wallaschofski $H$, Nauck M, et al. Ultrasonographic hepatic steatosis increases prediction of mortality risk from elevated serum gamma-glutamyl transpeptidase levels. Hepatology 2009;50:1403-11.

59 Hamaguchi M, Kojima T, Takeda N, et al. Nonalcoholic fatty liver disease is a novel predictor of cardiovascular disease. World J Gastroenterol 2007;13:1579-84.

60 Pisto P, Santaniemi M, Bloigu R, et al. Fatty liver predicts the risk for cardiovascular events in middle-aged population: a population-based cohort study. BMJ Open 2014:4:e004973.

61 Matteoni CA, Younossi ZM, Gramlich T, et al. Nonalcoholic fatty liver disease: a spectrum of clinical and pathological severity. Gastroenterology 1999;116: 1413-19.

62 Dam-Larsen S, Franzmann M, Andersen IB, et al. Long term prognosis of fatty liver: risk of chronic liver disease and death. Gut 2004;53:750-5.

63 Rafiq N, Bai C, Fang Y, et al. Long-term follow-up of patients with nonalcoholic fatty liver. Clin Gastroenterol Hepatol 2009;7:234-8.

64 Adams LA, Lymp JF, St Sauver J, et al. The natural history of nonalcoholic fatty liver disease: a population-based cohort study. Gastroenterology 2005;129:113-21.

65 Jepsen $\mathrm{P}$, Vilstrup $\mathrm{H}$, Møller JK, et al. Prognosis of patients with liver cirrhosis and spontaneous bacterial peritonitis. Hepatogastroenterology 2003;50:2133-6.

66 Targher G, Bertolini L, Rodella S, et al. Nonalcoholic fatty liver disease is independently associated with an increased incidence of cardiovascular events in type 2 diabetic patients. Diabetes Care 2007;30:2119-21.

67 Zhou YJ, Li YY, Nie YQ, et al. Natural course of nonalcoholic fatty liver disease in southern China: a prospective cohort study. J Dig Dis 2012;13:153-60.

68 Targher G, Byrne CD, Lonardo A, et al. Non-alcoholic fatty liver disease and risk of incident cardiovascular disease: a meta-analysis. J Hepatol 2016;65:589-600.

69 Anstee QM, Day CP. The genetics of nonalcoholic fatty liver disease: spotlight on PNPLA3 and TM6SF2. Semin Liver Dis 2015;35:270-90.

70 Romeo S, Kozlitina J, Xing C, et al. Genetic variation in PNPLA3 confers susceptibility to nonalcoholic fatty liver disease. Nat Genet 2008;40:1461-5.

71 Valenti L, Al-Serri A, Daly AK, et al. Homozygosity for the patatin-like phospholipase-3/adiponutrin $1148 \mathrm{M}$ polymorphism influences liver fibrosis in patients with nonalcoholic fatty liver disease. Hepatology 2010;51:1209-17.

72 Liu YL, Patman GL, Leathart JB, et al. Carriage of the PNPLA3 rs738409 C>G polymorphism confers an increased risk of non-alcoholic fatty liver disease associated hepatocellular carcinoma. J Hepatol 2014;61:75-81.

73 Lonardo A, Ballestri S, Targher G. "Not all forms of NAFLD were created equal". Do metabolic syndrome-related NAFLD and PNPLA3-related NAFLD exert a variable impact on the risk of early carotid atherosclerosis? Atherosclerosis 2017;257:253-5

74 Kozlitina J, Smagris E, Stender S, et al. Exome-wide association study identifies a TM6SF2 variant that confers susceptibility to nonalcoholic fatty liver disease. Nat Genet 2014;46:352-6.

75 Liu YL, Reeves HL, Burt AD, et al. TM6SF2 rs58542926 influences hepatic fibrosis progression in patients with non-alcoholic fatty liver disease. Nat Commun 2014;5:4309.

76 Dongiovanni P, Petta S, Maglio C, et al. Transmembrane 6 superfamily member 2 gene variant disentangles nonalcoholic steatohepatitis from cardiovascular disease. Hepatology 2015;61:506-14.

77 Holmen OL, Zhang H, Fan $\mathrm{Y}$, et al. Systematic evaluation of coding variation identifies a candidate causal variant in TM6SF2 influencing total cholesterol and myocardial infarction risk. Nat Genet 2014;46:345-51.

78 Kahali B, Liu YL, Daly AK, et al. TM6SF2: catch-22 in the fight against nonalcoholic fatty liver disease and cardiovascular disease? Gastroenterology 2015;148:679-84

79 Zeybel M, Hardy T, Wong YK, et al. Multigenerational epigenetic adaptation of the hepatic wound-healing response. Nat Med 2012;18:1369-77.

80 Zeybel M, Hardy T, Robinson SM, et al. Differential DNA methylation of genes involved in fibrosis progression in non-alcoholic fatty liver disease and alcoholic liver disease. Clin Epigenetics 2015;7:25.

81 Hardy T, Zeybel M, Day CP, et al. Plasma DNA methylation: a potential biomarker for stratification of liver fibrosis in non-alcoholic fatty liver disease. Gut 2016 doi: 10.1136/gutjnl-2016-311526. [Epub ahead of print 21 Mar 2016].

82 Ma Y, Ordovas JM. The integration of epigenetics and genetics in nutrition research for CVD risk factors. Proc Nutr Soc 2016:1-14.

83 Käräjämäki AJ, Pätsi OP, Savolainen $M$, et al. Non-alcoholic fatty liver disease as a predictor of atrial fibrillation in middle-aged population (OPERA study). PLOS One 2015; 10:e0142937

84 Targher G, Valbusa F, Bonapace S, et al. Non-alcoholic fatty liver disease is associated with an increased incidence of atrial fibrillation in patients with type 2 diabetes. PLoS One 2013;8:e57183

85 Mantovani A, Pernigo M, Bergamini $C$, et al. Heart valve calcification in patients with type 2 diabetes and nonalcoholic fatty liver disease. Metab Clin Exp 2015:64:879-87.

86 Hung CS, Tseng PH, Tu CH, et al. Nonalcoholic fatty liver disease is associated with QT prolongation in the general population. J Am Heart Assoc 2015;4: e001820.
87 Mantovani A, Rigamonti A, Bonapace $\mathrm{S}$, et al. Nonalcoholic fatty liver disease is associated with ventricular arrhythmias in patients with type 2 diabetes referred for clinically indicated 24-hour Holter monitoring. Diabetes Care 2016;39:1416-23.

88 Treeprasertsuk S, Leverage S, Adams LA, et al. The Framingham risk score and heart disease in nonalcoholic fatty liver disease. Liver Int 2012;32:945-50.

89 Targher G, Bertolini L, Rodella S, et al. Non-alcoholic fatty liver disease is independently associated with an increased prevalence of chronic kidney disease and proliferative/laser-treated retinopathy in type 2 diabetic patients. Diabetologia 2008;51:444-50.

90 Targher G, Bertolini L, Chonchol M, et al. Non-alcoholic fatty liver disease is independently associated with an increased prevalence of chronic kidney disease and retinopathy in type 1 diabetic patients. Diabetologia 2010;53:1341-8.

91 Li G, Shi W, Hug H, et al. Nonalcoholic fatty liver disease associated with impairment of kidney function in nondiabetes population. Biochem Med (Zagreb) 2012;22:92-9.

92 Sirota JC, McFann K, Targher G, et al. Association between nonalcoholic liver disease and chronic kidney disease: an ultrasound analysis from NHANES 19881994. Am J Nephrol 2012;36:466-71.

93 Ahn AL, Choi JK, Kim MN, et al. Non-alcoholic fatty liver disease and chronic kidney disease in Koreans aged 50 years or older. Korean J Fam Med 2013;34:199-205.

94 Mikolasevic I, Racki S, Bubic I, et al. Chronic kidney disease and nonalcoholic Fatty liver disease proven by transient elastography. Kidney Blood Press Res 2013:37:305-10.

95 Jia G, Di F, Wang Q, et al. Non-alcoholic fatty liver disease is a risk factor for the development of diabetic nephropathy in patients with type 2 diabetes mellitus. PLoS One 2015;10:e0142808.

96 Pan LL, Zhang HJ, Huang ZF, et al. Intrahepatic triglyceride content is independently associated with chronic kidney disease in obese adults: a cross-sectional study. Metab Clin Exp 2015;64:1077-85.

$97 \mathrm{Xu} \mathrm{HW}$, Hsu YC, Chang CH, et al. High FIB-4 index as an independent risk factor of prevalent chronic kidney disease in patients with nonalcoholic fatty liver disease. Hepatol Int 2016;10:340-6.

98 Pacifico L, Bonci E, Andreoli GM, et al. The impact of nonalcoholic fatty liver disease on renal function in children with overweight/obesity. Int J Mol Sci 2016;17.

99 Targher G, Bertolini L, Rodella S, et al. Relationship between kidney function and liver histology in subjects with nonalcoholic steatohepatitis. Clin J Am Soc Nephrol 2010;5:2166-71

100 Yilmaz Y, Alahdab YO, Yonal 0, et al. Microalbuminuria in nondiabetic patients with nonalcoholic fatty liver disease: association with liver fibrosis. Metab Clin Exp 2010:59:1327-30.

101 Yasui K, Sumida Y, Mori Y, et al. Nonalcoholic steatohepatitis and increased risk of chronic kidney disease. Metab Clin Exp 2011;60:735-9.

102 Park CW, Tsai NT, Wong LL. Implications of worse renal dysfunction and medical comorbidities in patients with NASH undergoing liver transplant evaluation: impact on MELD and more. Clin Transplant 2011;25:E606-11.

103 Machado MV, Gonçalves S, Carepa F, et al. Impaired renal function in morbid obese patients with nonalcoholic fatty liver disease. Liver Int 2012;32:241-8.

104 Lin L, Lu J, Huang X, et al. Nonalcoholic fatty liver disease is associated with low-grade albuminuria in Chinese adults (change not displayed). QJM 2016;109:737-43.

105 Musso G, Cassader M, Cohney S, et al. Fatty liver and chronic kidney disease: novel mechanistic insights and therapeutic opportunities. Diabetes Care 2016;39:1830-45.

106 Targher G, Chonchol MB, Byrne CD. CKD and nonalcoholic fatty liver disease. Am J Kidney Dis 2014;64:638-52.

107 Chang $Y$, Ryu S, Sung $E$, et al. Nonalcoholic fatty liver disease predicts chronic kidney disease in nonhypertensive and nondiabetic Korean men. Metab Clin Exp 2008:57:569-76.

108 Targher G, Chonchol M, Bertolini L, et al. Increased risk of CKD among type 2 diabetics with nonalcoholic fatty liver disease. J Am Soc Nephrol 2008; 19:1564-70

109 Targher G, Mantovani A, Pichiri I, et al. Nonalcoholic fatty liver disease is independently associated with an increased incidence of chronic kidney disease in patients with type 1 diabetes. Diabetes Care 2014;37:1729-36.

110 Musso G, Gambino R, Tabibian JH, et al. Association of non-alcoholic fatty liver disease with chronic kidney disease: a systematic review and meta-analysis. PLoS Med 2014;11:e1001680.

111 Vilar-Gomez E, Calzadilla-Bertot L, Friedman SL, et al. Improvement in liver histology due to lifestyle modification is independently associated with improved kidney function in patients with non-alcoholic steatohepatitis. Aliment Pharmacol Ther 2017:45:332-44.

112 Wong VW, Chu WC, Wong GL, et al. Prevalence of non-alcoholic fatty liver disease and advanced fibrosis in Hong Kong Chinese: a population study using protonmagnetic resonance spectroscopy and transient elastography. Gut 2012;61:409-15.

113 Browning JD, Szczepaniak LS, Dobbins R, et al. Prevalence of hepatic steatosis in an urban population in the United States: impact of ethnicity. Hepatology 2004;40:1387-95. 
114 Kwok R, Choi KC, Wong GL, et al. Screening diabetic patients for non-alcoholic fatty liver disease with controlled attenuation parameter and liver stiffness measurements: a prospective cohort study. Gut 2016;65:1359-68.

115 Fabbrini E, Yoshino J, Yoshino M, et al. Metabolically normal obese people are protected from adverse effects following weight gain. I Clin Invest 2015;125:787-95.

116 Perry IJ, Wannamethee SG, Shaper AG. Prospective study of serum gamma-glutamyltransferase and risk of NIDDM. Diabetes Care 1998:21:732-7.

117 Ballestri S, Zona S, Targher G, et al. Nonalcoholic fatty liver disease is associated with an almost twofold increased risk of incident type 2 diabetes and metabolic syndrome. Evidence from a systematic review and meta-analysis. J Gastroenterol Hepatol 2016;31:936-44.

118 Balkau B, Lange C, Vol S, et al. Group Study DESIR. Nine-year incident diabetes is predicted by fatty liver indices: the French D.E.S.I.R. study. BMC Gastroenterol 2010;10:56.

119 Chang Y, Jung HS, Yun KE, et al. Cohort study of non-alcoholic fatty liver disease, NAFLD fibrosis score, and the risk of incident diabetes in a Korean population. Am J Gastroenterol 2013;108:1861-8.

120 Chen GY, Cao HX, Li F, et al. New risk-scoring system including non-alcoholic fatty liver disease for predicting incident type 2 diabetes in East China: Shanghai Baosteel Cohort. J Diabetes Investig 2016;7:206-11.

121 Fukuda T, Hamaguchi M, Kojima T, et al. The impact of non-alcoholic fatty liver disease on incident type 2 diabetes mellitus in non-overweight individuals. Liver Int 2016:36:275-83.

$122 \mathrm{Kim} \mathrm{CH}$, Park JY, Lee KU, et al. Fatty liver is an independent risk factor for the development of Type 2 diabetes in Korean adults. Diabet Med 2008:25:476-81.

123 Ming J, Xu S, Gao B, et al. Non-alcoholic fatty liver disease predicts type 2 diabetes mellitus, but not prediabetes, in Xi'an, China: a five-year cohort study. Liver Int 2015;35:2401-7.

124 Okamoto M, Takeda Y, Yoda Y, et al. The association of fatty liver and diabetes risk. J Epidemiol 2003;13:15-21.

125 Park SK, Seo MH, Shin HC, et al. Clinical availability of nonalcoholic fatty liver disease as an early predictor of type 2 diabetes mellitus in Korean men: 5-year prospective cohort study. Hepatology 2013;57:1378-83.

126 Shah RV, Allison MA, Lima JA, et al. Liver fat, statin use, and incident diabetes: The Multi-Ethnic Study of Atherosclerosis. Atherosclerosis 2015;242:211-17.

127 Sung KC, Kim SH. Interrelationship between fatty liver and insulin resistance in the development of type 2 diabetes. J Clin Endocrinol Metab 2011;96:1093-7.

128 Yamada T, Fukatsu M, Suzuki S, et al. Fatty liver predicts impaired fasting glucose and type 2 diabetes mellitus in Japanese undergoing a health checkup. J Gastroenterol Hepatol 2010;25:352-6.

129 Yamazaki H, Tsuboya T, Tsuji K, et al. Independent association between improvement of nonalcoholic fatty liver disease and reduced incidence of type 2 diabetes. Diabetes Care 2015;38:1673-9.

130 Sung KC, Wild SH, Byrne CD. Resolution of fatty liver and risk of incident diabetes. I Clin Endocrinol Metab 2013:98:3637-43.

131 Fukuda T, Hamaguchi M, Kojima T, et al. Transient remission of nonalcoholic fatty liver disease decreases the risk of incident type 2 diabetes mellitus in Japanese men. Eur J Gastroenterol Hepatol 2016;28:1443-9.

132 Sung KC, Jeong WS, Wild SH, et al. Combined influence of insulin resistance, overweight/obesity, and fatty liver as risk factors for type 2 diabetes. Diabetes Care 2012;35:717-22

133 Bae JC, Rhee EJ, Lee WY, et al. Combined effect of nonalcoholic fatty liver disease and impaired fasting glucose on the development of type 2 diabetes: a 4-year retrospective longitudinal study. Diabetes Care 2011:34:727-9.

134 Käräjämäki AJ, Bloigu R, Kauma $H$, et al. Non-alcoholic fatty liver disease with and without metabolic syndrome: different long-term outcomes. Metab Clin Exp 2017;66:55-63.

135 Choi JH, Rhee EJ, Bae JC, et al. Increased risk of type 2 diabetes in subjects with both elevated liver enzymes and ultrasonographically diagnosed nonalcoholic fatty liver disease: a 4-year longitudinal study. Arch Med Res 2013:44:115-20

136 Lin TY, Chen YJ, Chen WL, et al. The relationship between nonalcoholic fatty liver disease and retinopathy in NHANES III. PLoS One 2016;11:e0165970.

137 Amaro A, Fabbrini E, Kars $M$, et al. Dissociation between intrahepatic triglyceride content and insulin resistance in familial hypobetalipoproteinemia. Gastroenterology 2010;139:149-53.

138 Palmer CN, Maglio C, Pirazzi C, et al. Paradoxical lower serum triglyceride levels and higher type 2 diabetes mellitus susceptibility in obese individuals with the PNPLA3 148M variant. PLOS ONE 2012;7:e39362.

139 European Association for the Study of the Liver (EASL); European Association for the Study of Diabetes (EASD); European Association for the Study of Obesity (EASO). EASL-EASD-EASO Clinical Practice Guidelines for the management of non-alcoholic fatty liver disease. J Hepatol 2016;64:1388-402.

140 Adams LA, Harmsen S, St Sauver JL, et al. Nonalcoholic fatty liver disease increases risk of death among patients with diabetes: a community-based cohort study. Am J Gastroenterol 2010;105:1567-73.
141 Huang KW, Leu HB, Wang YJ, et al. Patients with nonalcoholic fatty liver disease have higher risk of colorectal adenoma after negative baseline colonoscopy. Colorectal Dis 2013;15:830-5.

142 Ding W, Fan J, Qin J. Association between nonalcoholic fatty liver disease and colorectal adenoma: a systematic review and meta-analysis. Int J Clin Exp Med 2015:8:322-33.

143 Shen $\mathrm{H}$, Lipka S, Kumar A, et al. Association between nonalcoholic fatty liver disease and colorectal adenoma: a systemic review and meta-analysis. J Gastrointest Oncol 2014;5:440-6.

144 Wong VW, Wong GL, Tsang SW, et al. High prevalence of colorectal neoplasm in patients with non-alcoholic steatohepatitis. Gut 2011;60:829-36.

145 Touzin NT, Bush KN, Williams CD, et al. Prevalence of colonic adenomas in patients with nonalcoholic fatty liver disease. Therap Adv Gastroenterol 2011:4:169-76.

146 Ahn JS, Sinn DH, Min YW, et al. Non-alcoholic fatty liver diseases and risk of colorectal neoplasia. Aliment Pharmacol Ther 2017;45:345-53.

147 Lee YI, Lim YS, Park HS. Colorectal neoplasms in relation to non-alcoholic fatty liver disease in Korean women: a retrospective cohort study. J Gastroenterol Hepatol 2012;27:91-5.

148 Lin XF, Shi KQ, You J, et al. Increased risk of colorectal malignant neoplasm in patients with nonalcoholic fatty liver disease: a large study. Mol Biol Rep 2014;41:2989-97.

149 Sanna C, Rosso C, Marietti M, et al. Non-alcoholic fatty liver disease and extra-hepatic cancers. Int J Mo/ Sci 2016;17:E717.

150 Targher G, Lonardo A, Rossini M. Nonalcoholic fatty liver disease and decreased bone mineral density: is there a link? J Endocrinol Invest 2015;38:817-25.

151 Pardee PE, Dunn W, Schwimmer JB. Non-alcoholic fatty liver disease is associated with low bone mineral density in obese children. Aliment Pharmacol Ther 2012;35:248-54.

152 Upala S, Jaruvongvanich V, Wijarnpreecha K, et al. Nonalcoholic fatty liver disease and osteoporosis: a systematic review and meta-analysis. J Bone Miner Metab 2016.

153 Xia MF, Lin HD, Yan HM, et al. The association of liver fat content and serum alanine aminotransferase with bone mineral density in middle-aged and elderly Chinese men and postmenopausal women. J Trans/ Med 2016;14:11

154 Lee SH, Yun JM, Kim SH, et al. Association between bone mineral density and nonalcoholic fatty liver disease in Korean adults. J Endocrinol Invest 2016:39:1329-36.

155 Yang HJ, Shim SG, Ma BO, et al. Association of nonalcoholic fatty liver disease with bone mineral density and serum osteocalcin levels in Korean men. Eur J Gastroenterol Hepatol 2016;28:338-44.

156 Li M, Xu Y, Xu M, et al. Association between nonalcoholic fatty liver disease (NAFLD) and osteoporotic fracture in middle-aged and elderly Chinese. J Clin Endocrinol Metab 2012;97:2033-8.

157 Gregor MF, Hotamisligil GS. Inflammatory mechanisms in obesity. Annu Rev Immunol 2011;29:415-45.

158 Pihlajämaki J, Kuulasmaa T, Kaminska D, et al. Serum interleukin 1 receptor antagonist as an independent marker of non-alcoholic steatohepatitis in humans. J Hepatol 2012;56:663-70.

159 Tilg H, Moschen AR, Szabo G. Interleukin-1 and inflammasomes in alcoholic liver disease/acute alcoholic hepatitis and nonalcoholic fatty liver disease/nonalcoholic steatohepatitis. Hepatology 2016:64:955-65.

160 Johnson AM, Olefsky JM. The origins and drivers of insulin resistance. Cell 2013:152:673-84.

161 Tilg $H$, Moschen AR. Evolution of inflammation in nonalcoholic fatty liver disease: the multiple parallel hits hypothesis. Hepatology 2010;52:1836-46.

162 Mohamed-Ali V, Goodrick S, Rawesh A, et al. Subcutaneous adipose tissue releases interleukin-6, but not tumor necrosis factor-alpha, in vivo. J Clin Endocrinol Metab 1997;82:4196-200.

163 Mottillo EP, Desjardins EM, Crane JD, et al. Lack of adipocyte AMPK exacerbates insulin resistance and hepatic steatosis through brown and beige adipose tissue function. Cell Metab 2016;24:118-29.

164 Softic S, Boucher J, Solheim MH, et al. Lipodystrophy due to adipose tissue-specific insulin receptor knockout results in progressive NAFLD. Diabetes 2016:65:2187-200.

165 Wieser V, Adolph TE, Grander C, et al. Adipose type I interferon signalling protects against metabolic dysfunction. Gut 2016. doi: 10.1136/gutjnl-2016-313155. [Epub ahead of print 23 Dec 2016].

166 Kau AL, Ahern PP, Griffin NW, et al. Human nutrition, the gut microbiome and the immune system. Nature 2011;474:327-36.

167 Tilg H, Moschen AR. Food, immunity, and the microbiome. Gastroenterology 2015:148:1107-19.

168 Kleinewietfeld M, Manzel A, Titze J, et al. Sodium chloride drives autoimmune disease by the induction of pathogenic TH17 cells. Nature 2013:496:518-22.

169 Calder PC. Fatty acids and inflammation: the cutting edge between food and pharma. Eur J Pharmacol 2011;668(Suppl 1):S50-8. 
170 Bischoff SC, Barbara G, Buurman W, et al. Intestinal permeability-a new target for disease prevention and therapy. BMC Gastroenterol 2014;14: 189.

171 Rahman K, Desai C, lyer SS, et al. Loss of junctional adhesion molecule a promotes severe steatohepatitis in mice on a diet high in saturated fat, fructose, and cholesterol. Gastroenterology 2016;151:733-46.e12.

172 Pendyala S, Walker JM, Holt PR. A high-fat diet is associated with endotoxemia that originates from the gut. Gastroenterology 2012;142:1100-1.e2.

173 Devkota S, Wang Y, Musch MW, et al. Dietary-fat-induced taurocholic acid promotes pathobiont expansion and colitis in $1110-1-$ mice. Nature 2012:487:104-8

174 Wang Z, Klipfell E, Bennett BJ, et al. Gut flora metabolism of phosphatidylcholine promotes cardiovascular disease. Nature 2011;472:57-63.

175 Jegatheesan $\mathrm{P}$, Beutheu S, Freese K, et al. Preventive effects of citrulline on Western diet-induced non-alcoholic fatty liver disease in rats. Br J Nutr 2016;116:191-203

176 Tilg H, Cani PD, Mayer EA. Gut microbiome and liver diseases. Gut 2016;65:2035-44.

177 Del Chierico F, Nobili V, Vernocchi $\mathrm{P}$, et al. Gut microbiota profiling of pediatric NAFLD and obese patients unveiled by an integrated meta-omics based approach. Hepatology 2017;65:451-64.

178 Boursier J, Mueller O, Barret $\mathrm{M}$, et al. The severity of nonalcoholic fatty liver disease is associated with gut dysbiosis and shift in the metabolic function of the gut microbiota. Hepatology 2016;63:764-75.

179 Yoshimoto S, Loo TM, Atarashi K, et al. Obesity-induced gut microbial metabolite promotes liver cancer through senescence secretome. Nature 2013:499:97-101.

180 Feng $\mathrm{Q}$, Liang $\mathrm{S}$, Jia $\mathrm{H}$, et al. Gut microbiome development along the colorectal adenoma-carcinoma sequence. Nat Commun 2015:6:6528.

181 Tilg H, Diehl AM. Cytokines in alcoholic and nonalcoholic steatohepatitis. N Engl J Med 2000;343:1467-76.

182 Cai D, Yuan M, Frantz DF, et al. Local and systemic insulin resistance resulting from hepatic activation of IKK-beta and NF-kappaB. Nat Med 2005;11:183-90.

183 Arkan MC, Hevener AL, Greten FR, et al. IKK-beta links inflammation to obesity-induced insulin resistance. Nat Med 2005;11:191-8.

184 Kiechl S, Wittmann J, Giaccari A, et al. Blockade of receptor activator of nuclear factor- $\kappa B$ (RANKL) signaling improves hepatic insulin resistance and prevents development of diabetes mellitus. Nat Med 2013;19:358-63.

185 Duparc T, Plovier H, Marrachelli VG, et al. Hepatocyte MyD88 affects bile acids, gut microbiota and metabolome contributing to regulate glucose and lipid metabolism. Gut 2016. doi: gutjnl-2015-310904. [Epub ahead of print 5 May 2016].

186 Zoller $\mathrm{H}$, Tilg H. Nonalcoholic fatty liver disease and hepatocellular carcinoma. Metab Clin Exp 2016:65:1151-60.

187 Park EJ, Lee JH, Yu GY, et al. Dietary and genetic obesity promote liver inflammation and tumorigenesis by enhancing IL-6 and TNF expression. Cell 2010;140:197-208.

188 Kaser S, Moschen A, Cayon A, et al. Adiponectin and its receptors in non-alcoholic steatohepatitis. Gut 2005;54:117-21.

189 Tilg H, Moschen AR. Adipocytokines: mediators linking adipose tissue, inflammation and immunity. Nat Rev Immunol 2006;6:772-83.

190 Wei EK, Giovannucci E, Fuchs CS, et al. Low plasma adiponectin levels and risk of colorectal cancer in men: a prospective study. I Natl Cancer Inst 2005; 97:1688-94

191 Yamaji T, Iwasaki M, Sasazuki S, et al. Interaction between adiponectin and leptin influences the risk of colorectal adenoma. Cancer Res 2010;70:5430-7.

192 Inamura K, Song M, Jung S, et al. Prediagnosis plasma adiponectin in relation to colorectal cancer risk according to KRAS mutation status. J Natl Cancer Inst 2016;108.

193 Fracanzani AL, Tiraboschi S, Pisano G, et al. Progression of carotid vascular damage and cardiovascular events in non-alcoholic fatty liver disease patients compared to the general population during 10 years of follow-up. Atherosclerosis 2016;246:208-13.

194 Mantovani A, Mingolla L, Rigolon R, et al. Nonalcoholic fatty liver disease is independently associated with an increased incidence of cardiovascular disease in adult patients with type 1 diabetes. Int J Cardiol 2016;225:387-91.

195 Zeb I, Li D, Budoff MJ, et al. Nonalcoholic fatty liver disease and incident cardiac events: the multi-ethnic study of atherosclerosis. J Am Coll Cardiol 2016;67:1965-6.

196 Hwang ST, Cho YK, Park JH, et al. Relationship of non-alcoholic fatty liver disease to colorectal adenomatous polyps. J Gastroenterol Hepatol 2010;25:562-7.

197 Stadlmayr A, Aigner E, Steger B, et al. Nonalcoholic fatty liver disease: an independent risk factor for colorectal neoplasia. J Intern Med 2011;270:41-9.

198 Bhatt BD, Lukose T, Siegel AB, et al. Increased risk of colorectal polyps in patients with non-alcoholic fatty liver disease undergoing liver transplant evaluation. J Gastrointest Oncol 2015;6:459-68.

199 Basyigit S, Uzman M, Kefeli A, et al. Absence of non-alcoholic fatty liver disease in the presence of insulin resistance is a strong predictor for colorectal carcinoma. Int J Clin Exp Med 2015:8:18601-10. 